\title{
InceptionV3-based model
}

\section{$1 \times 5$-Fold Cross-Validation}

Learning curves

Fold1

Training Loss and Accuracy on COVID-19 Dataset

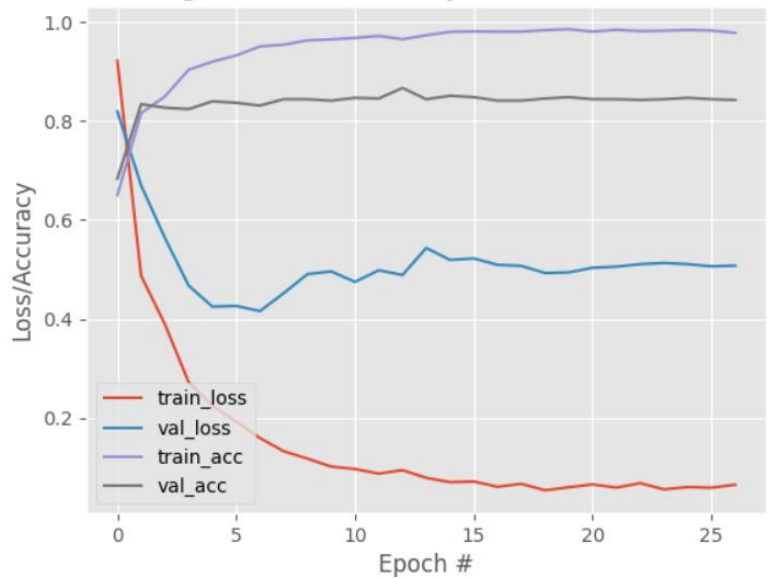

Fold4

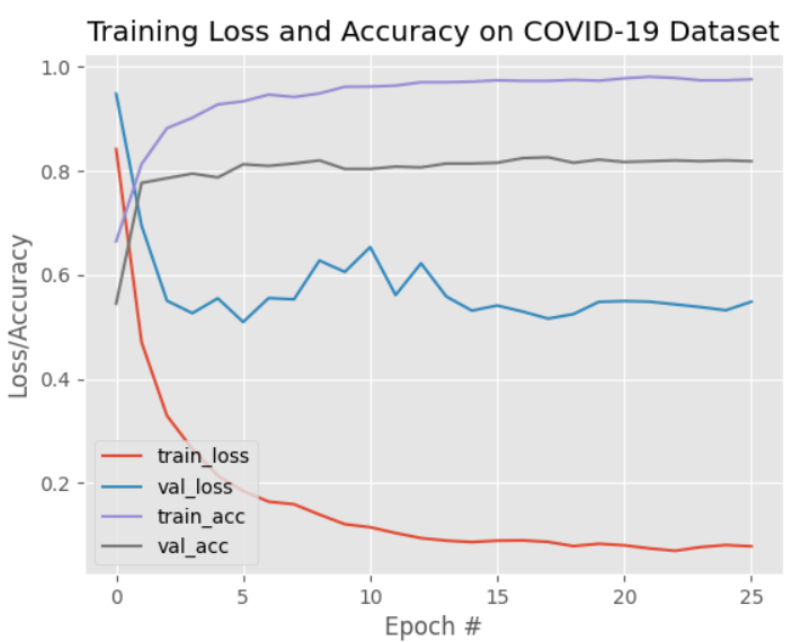

Fold2

Training Loss and Accuracy on COVID-19 Dataset

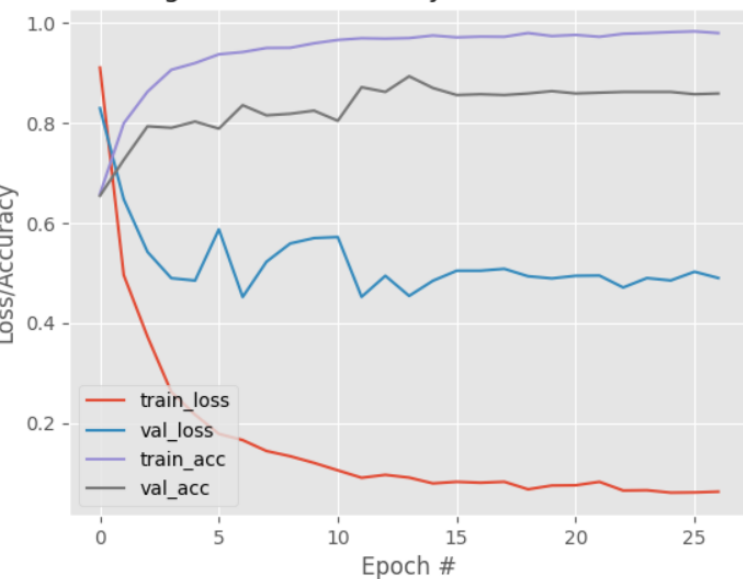

Fold5

Training Loss and Accuracy on COVID-19 Dataset

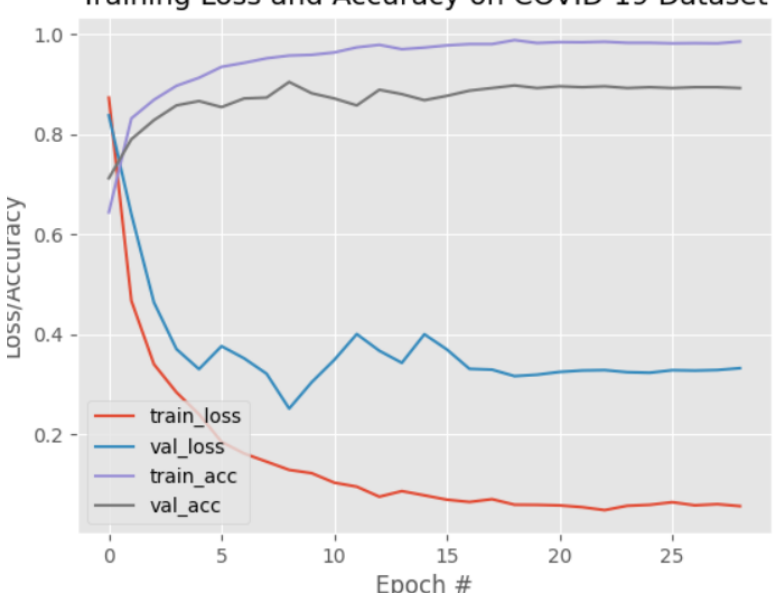

Fold3

Training Loss and Accuracy on COVID-19 Dataset

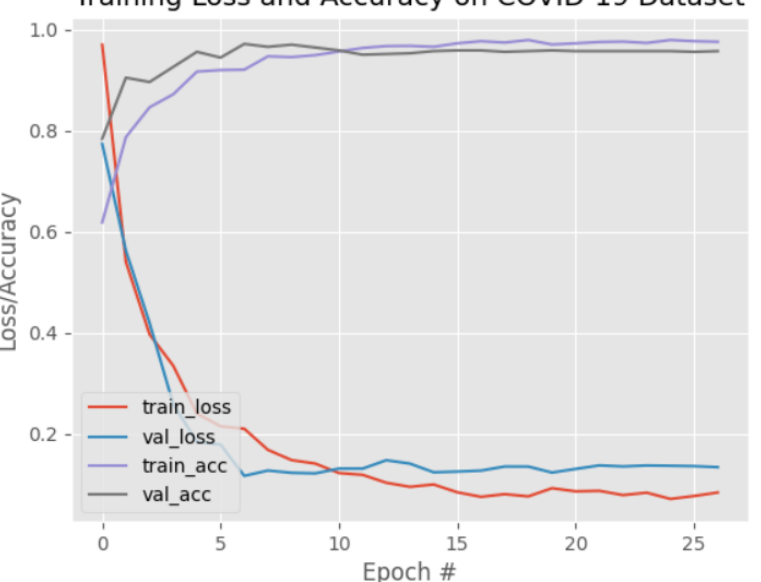




\section{Confusion matrices}

Fold1

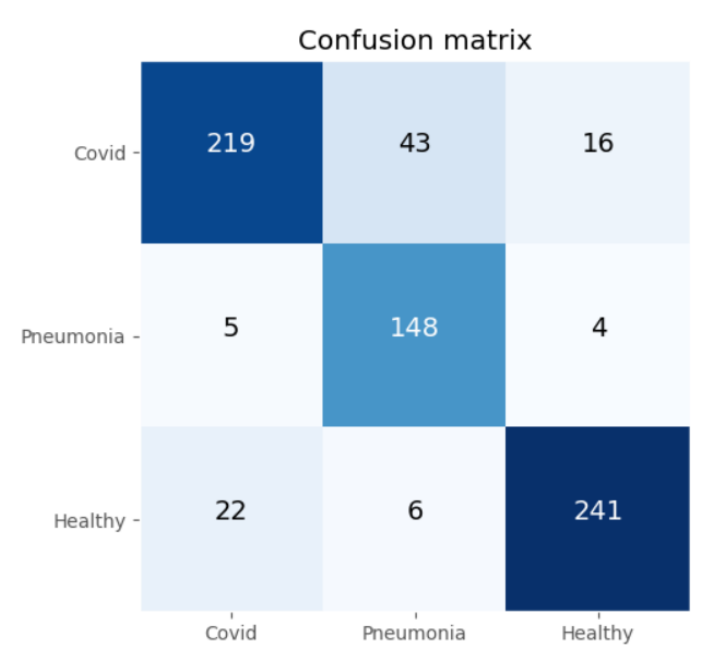

Fold4

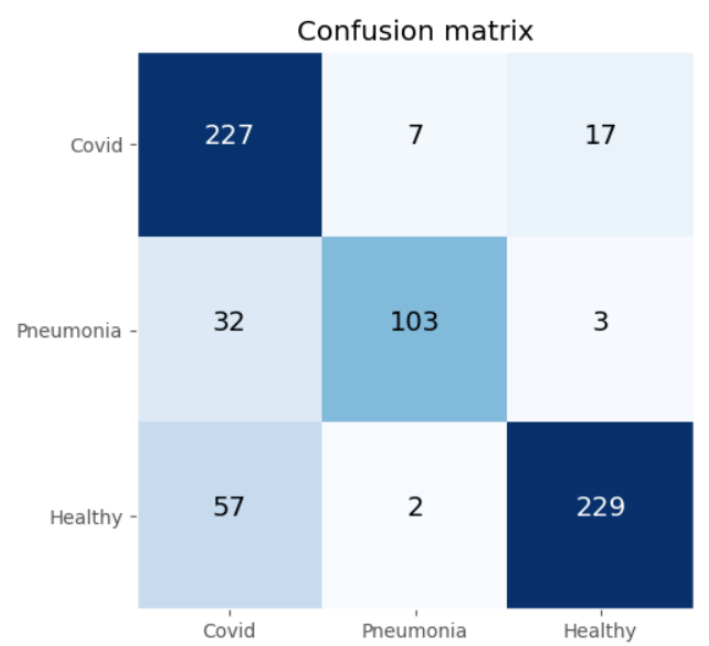

Fold2
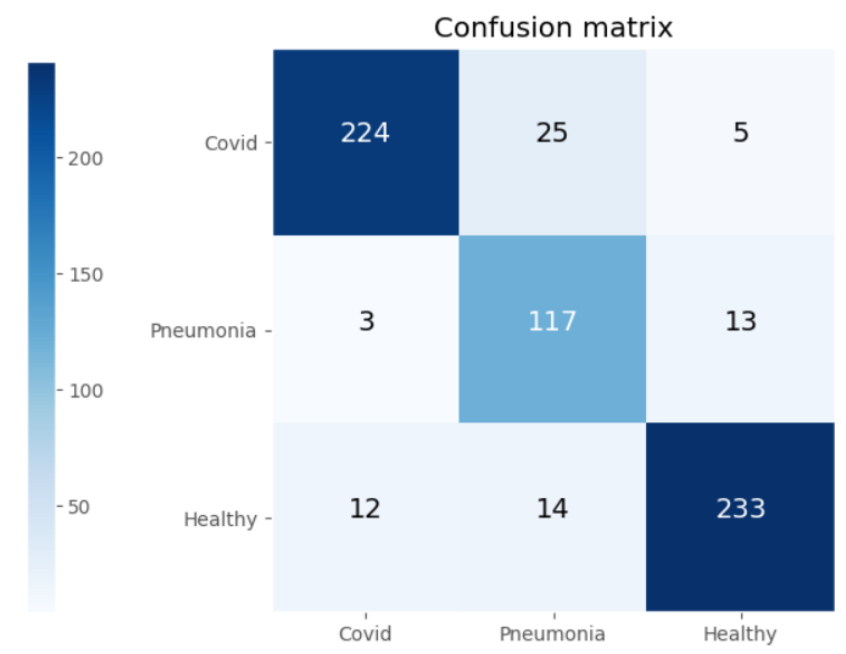

Fold5
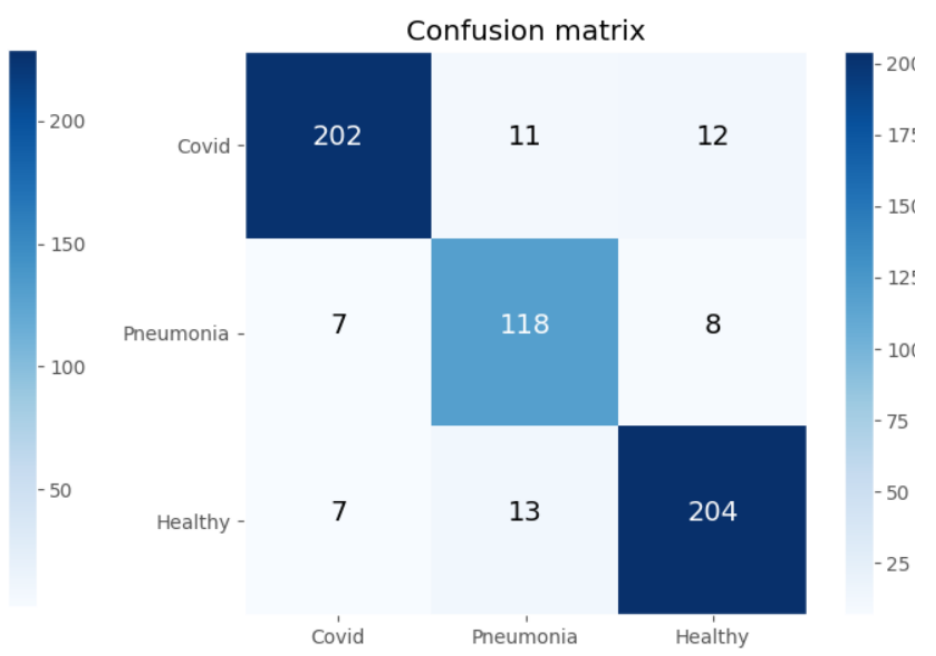

Fold3

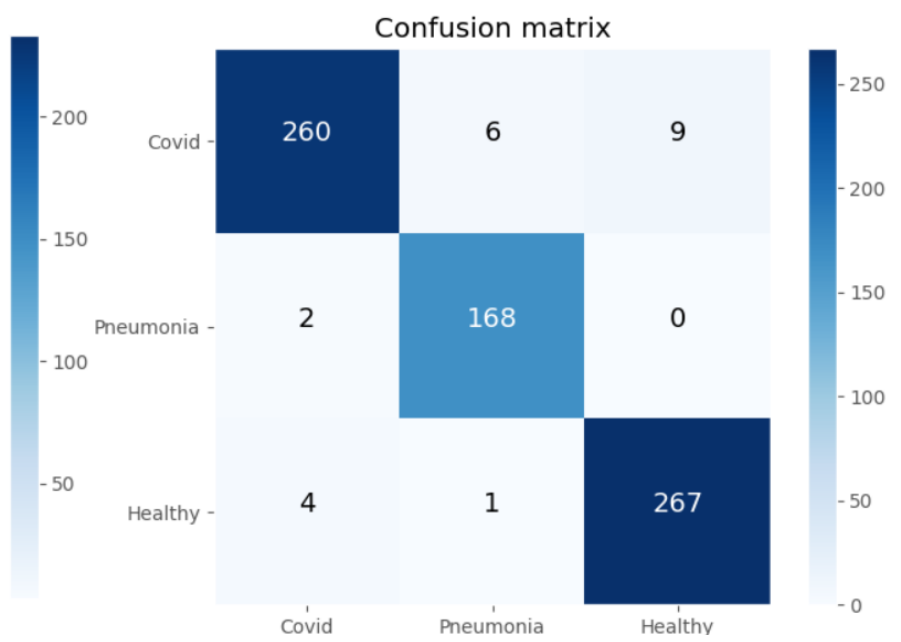

Healthy 
Fold1

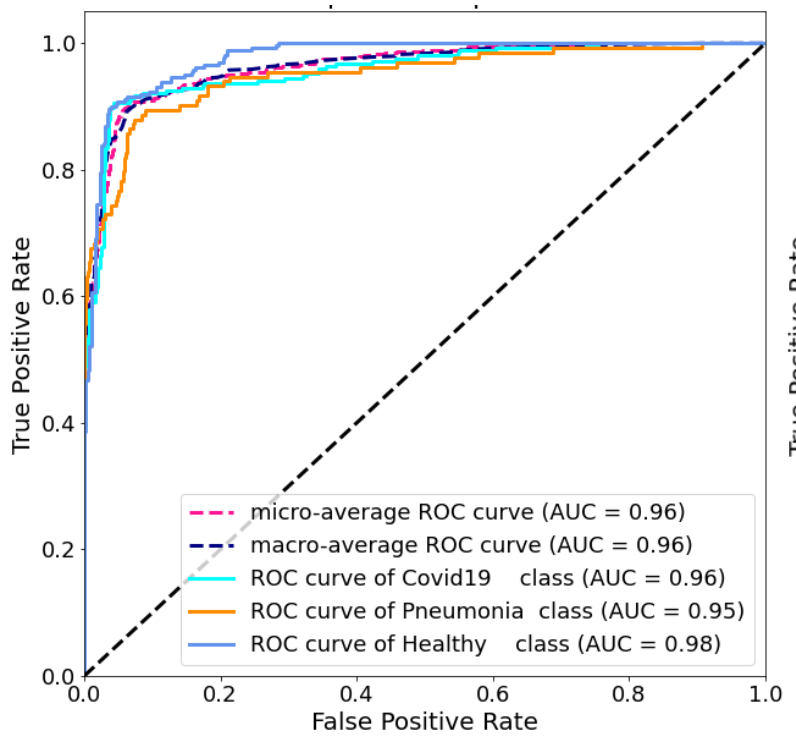

Fold4

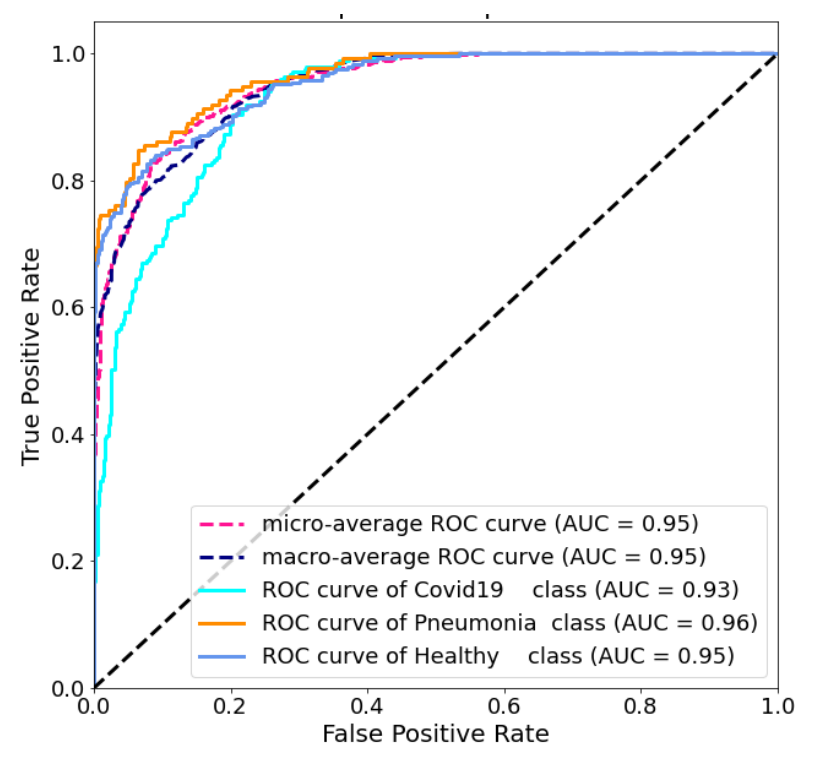

Fold2

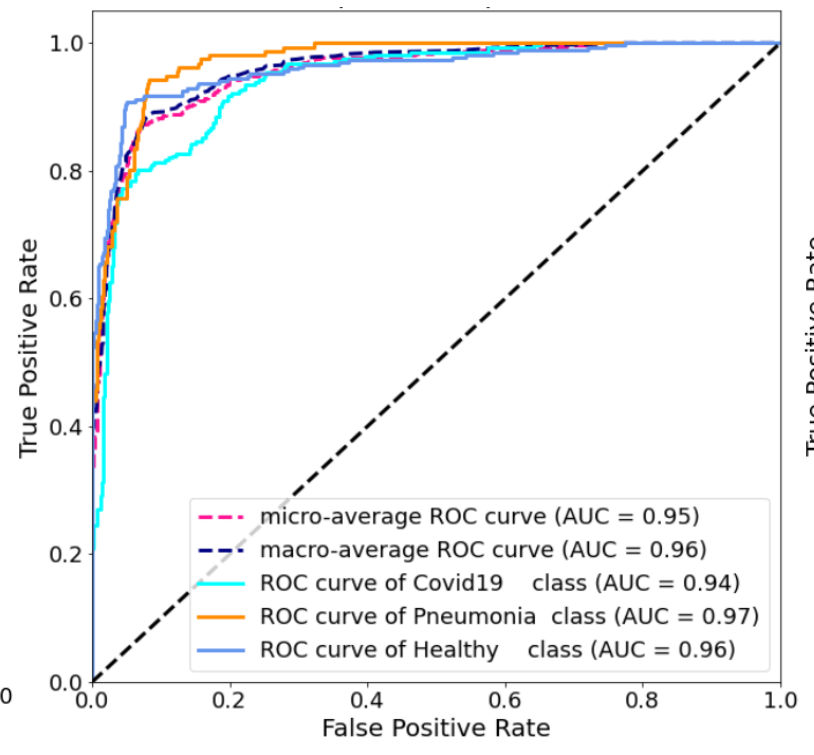

Fold5

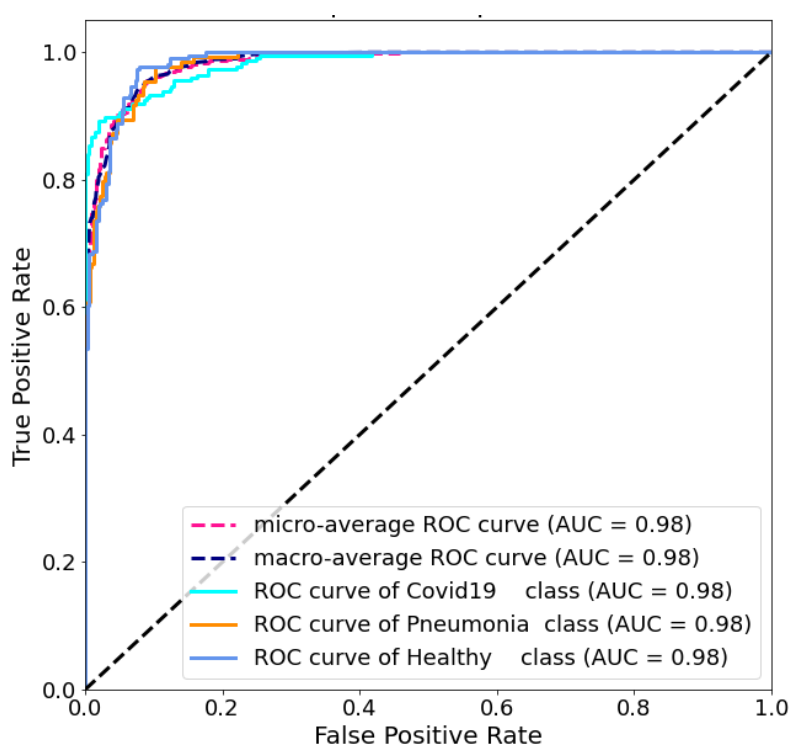

Fold3

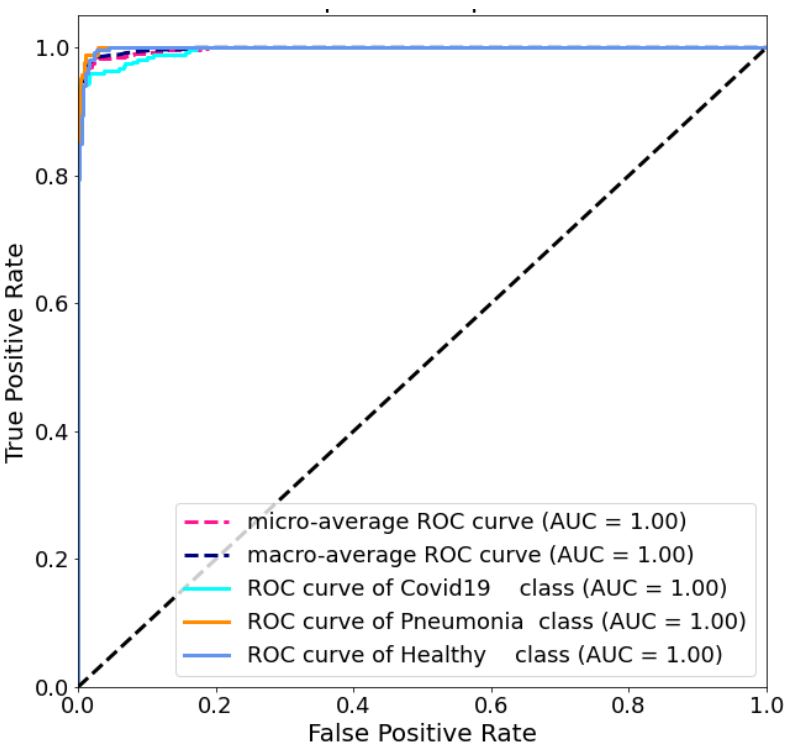




\section{$2 \times 5$-Fold Cross-Validation}

\section{Learning curves}

Fold1

Training Loss and Accuracy on COVID-19 Dataset

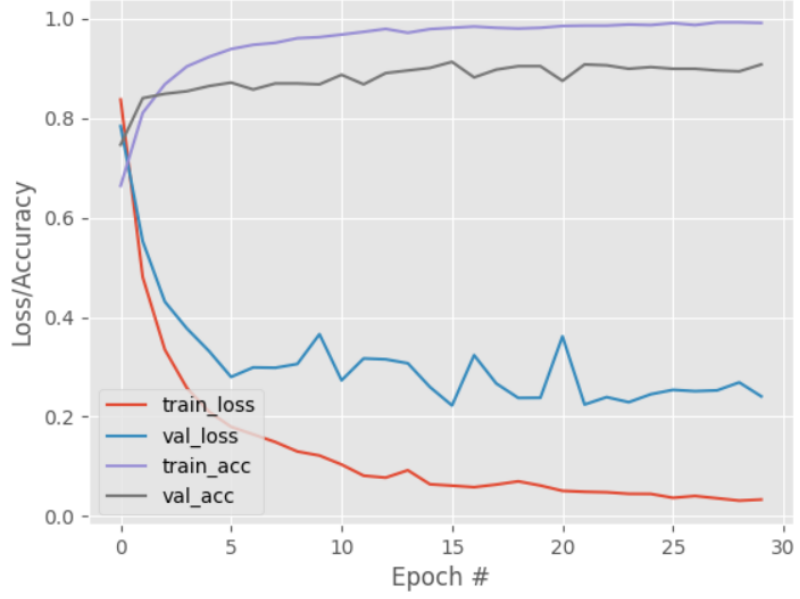

Fold4

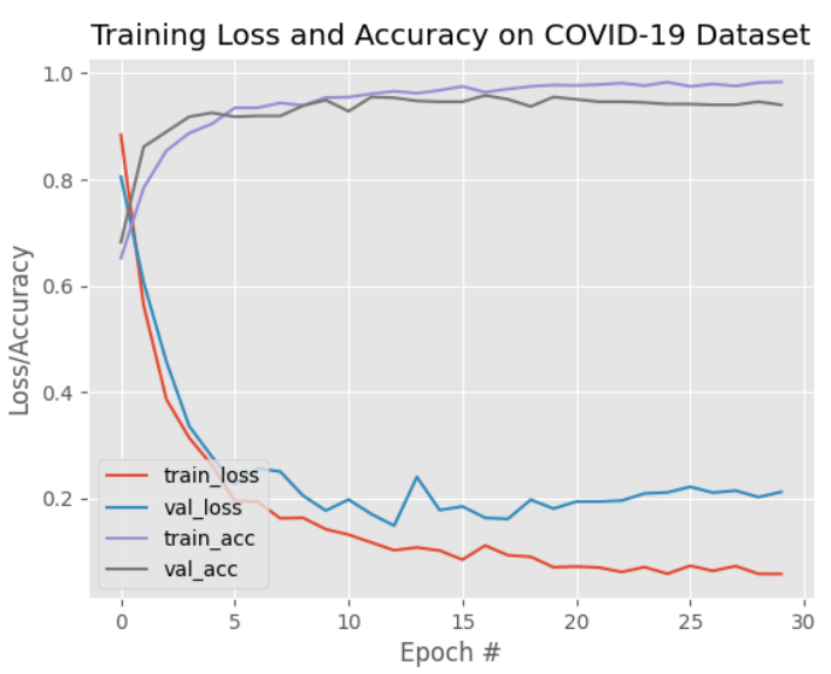

Fold2

Training Loss and Accuracy on COVID-19 Dataset

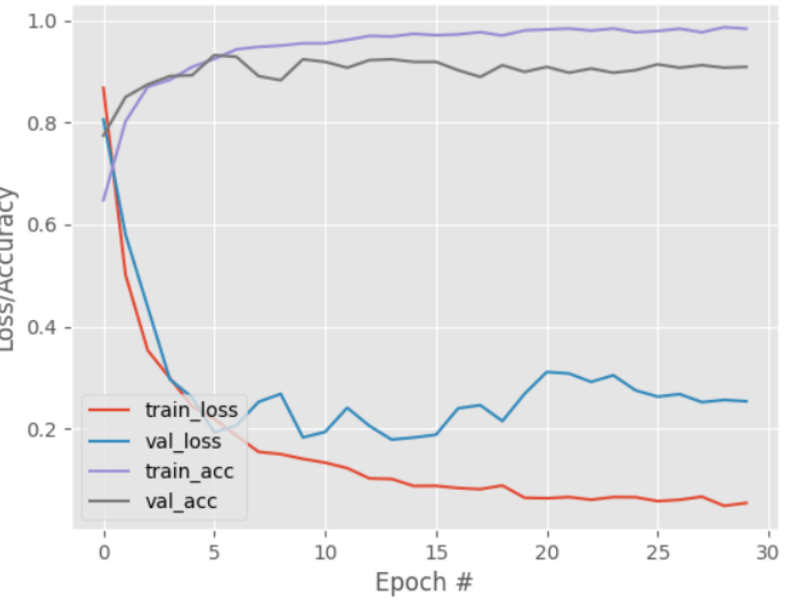

Fold5

Training Loss and Accuracy on COVID-19 Dataset

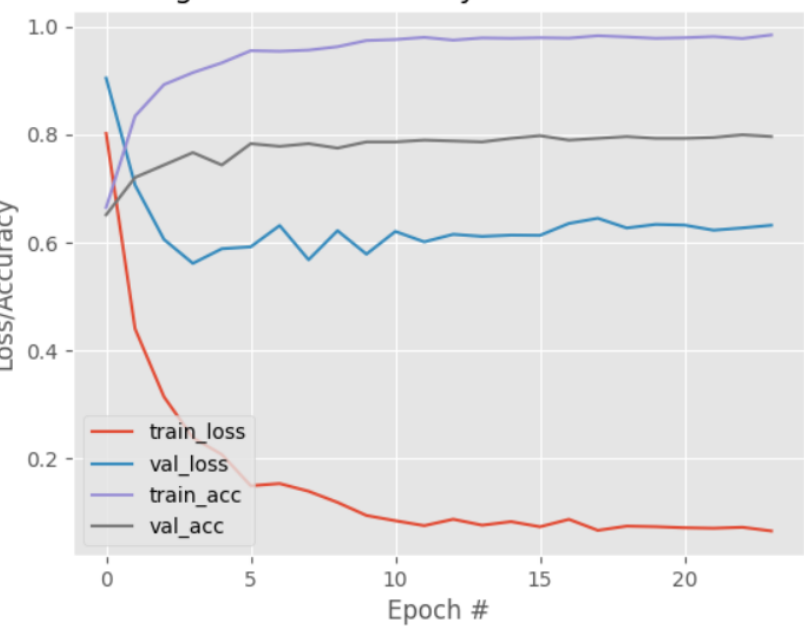

Fold3

Training Loss and Accuracy on COVID-19 Dataset

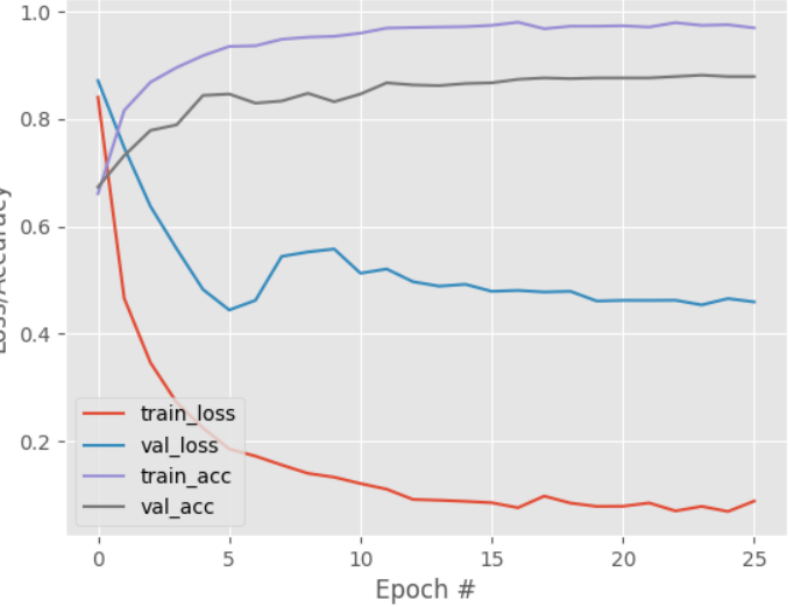




\section{Confusion matrices}

Fold1

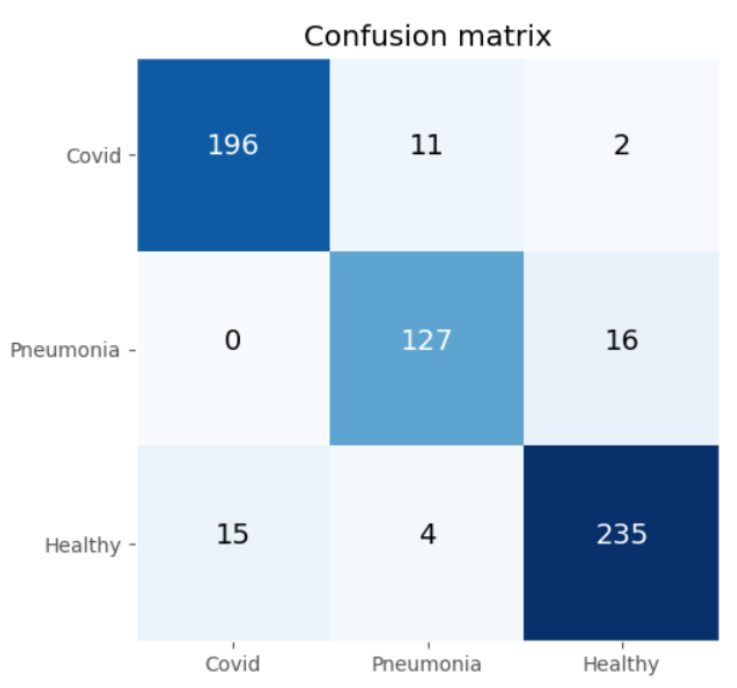

Fold4

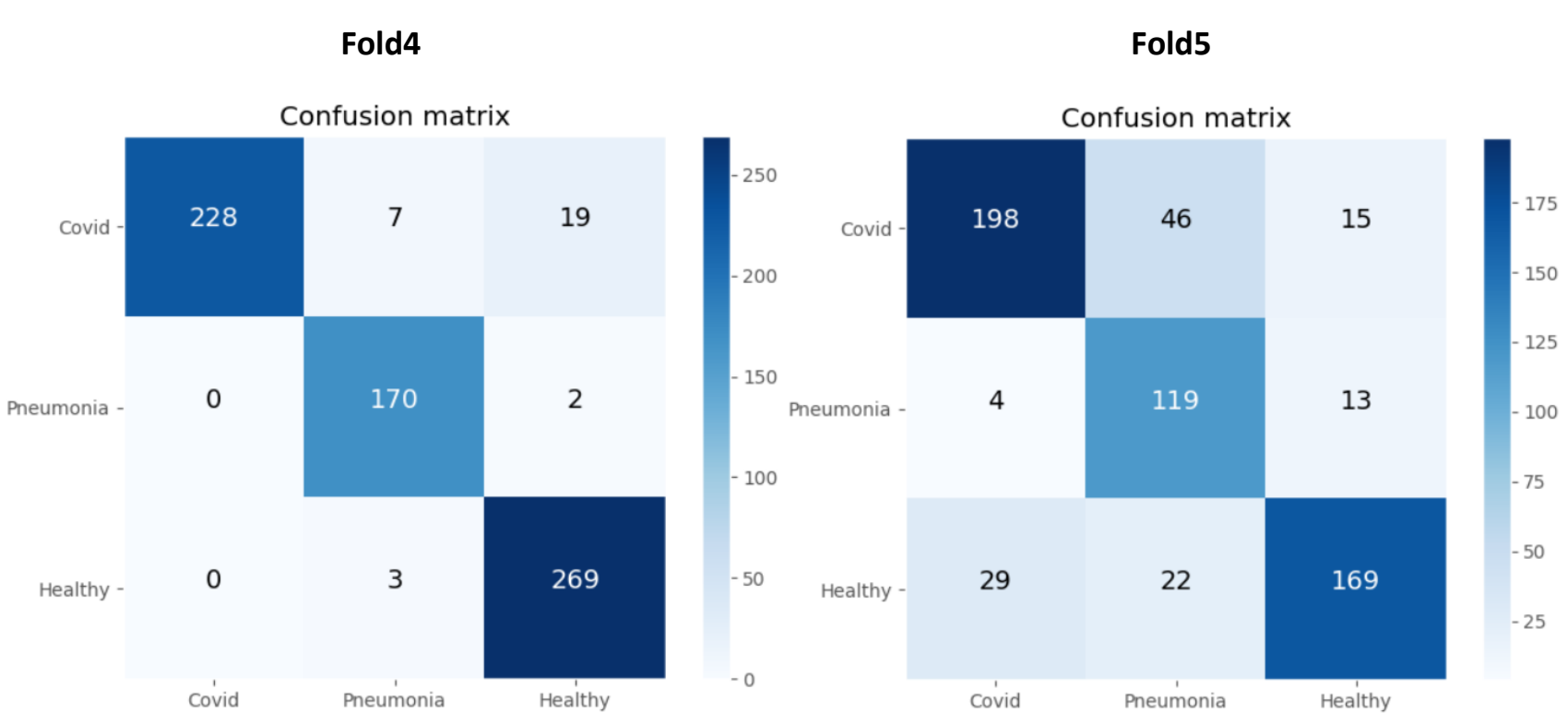

Fold2

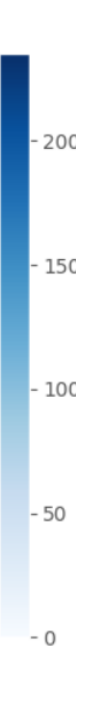

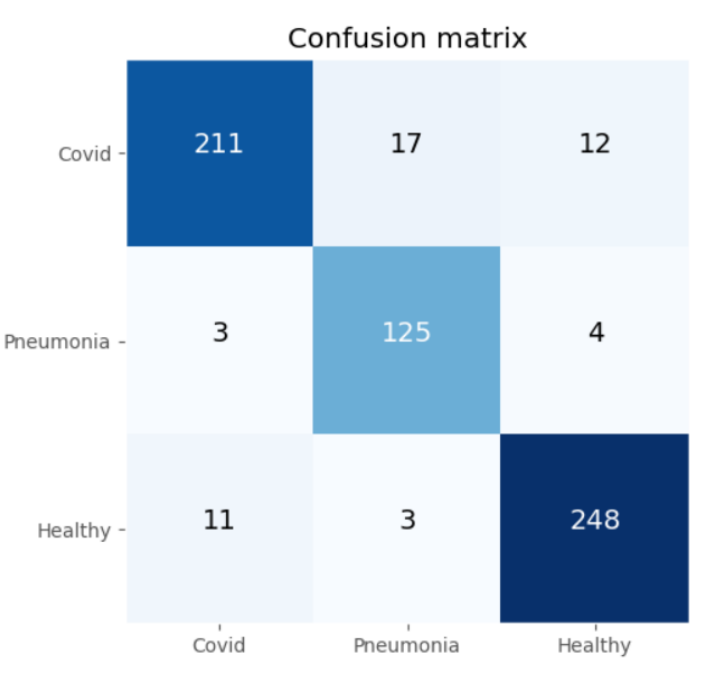
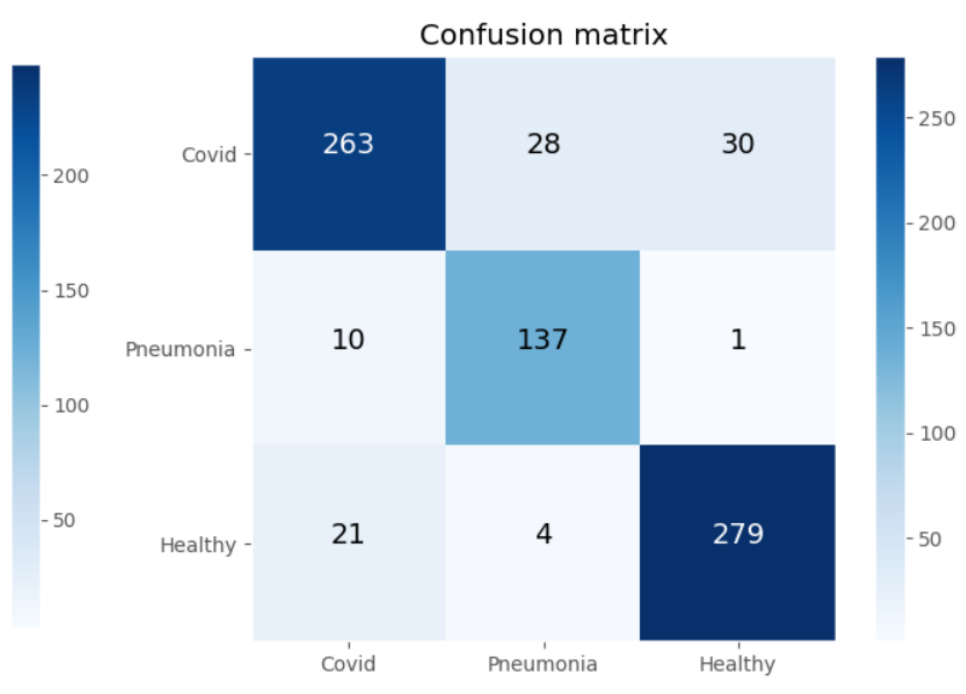

Fold5

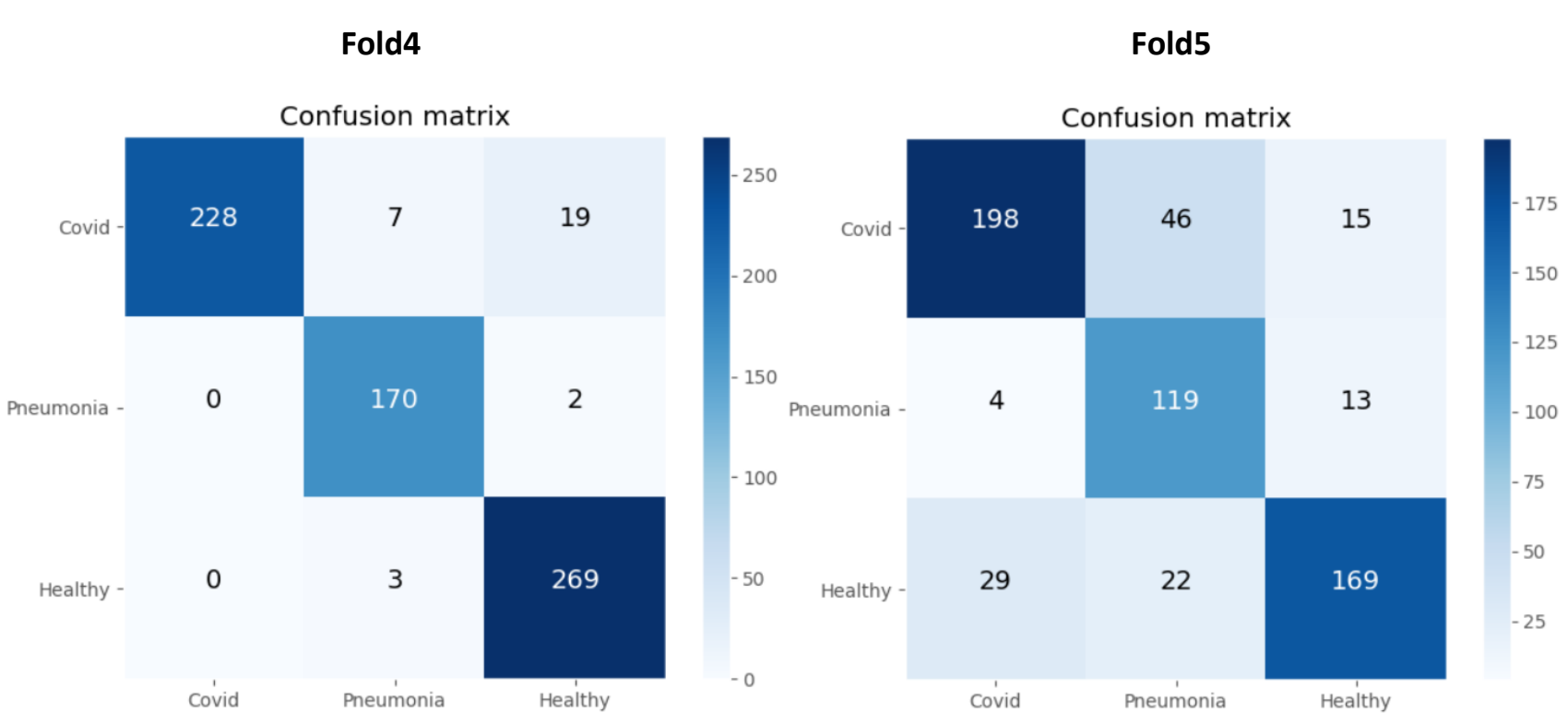

Fold3 


\section{ROC curves}

Fold1

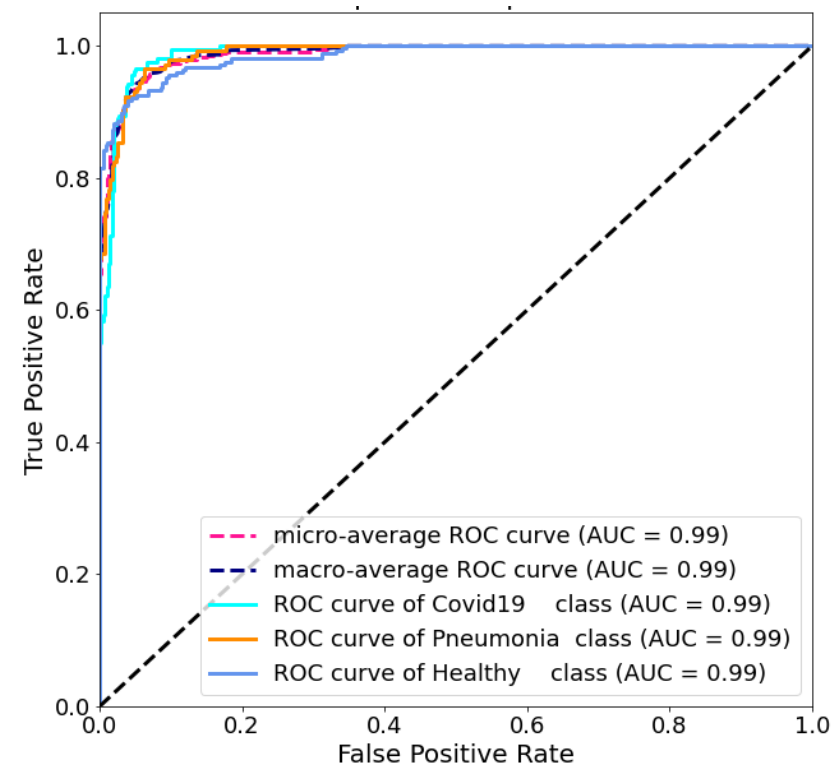

Fold4

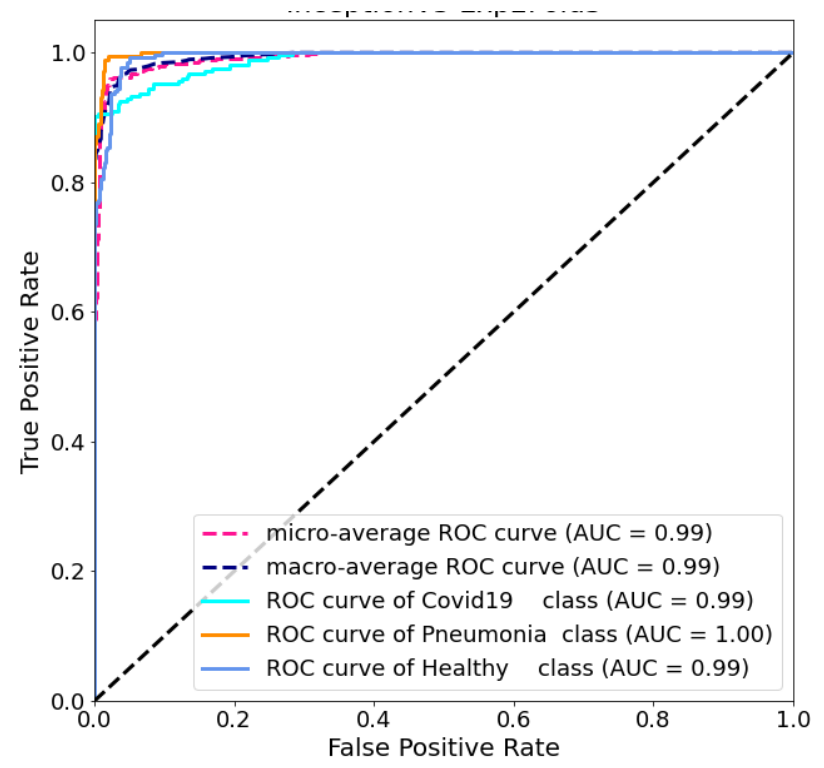

Fold2

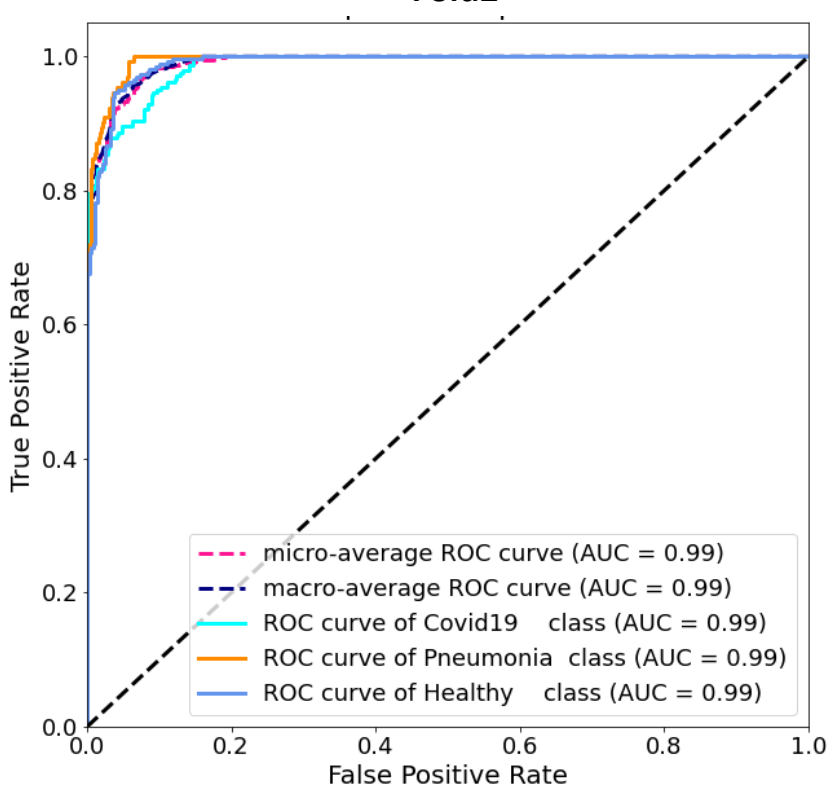

Fold5

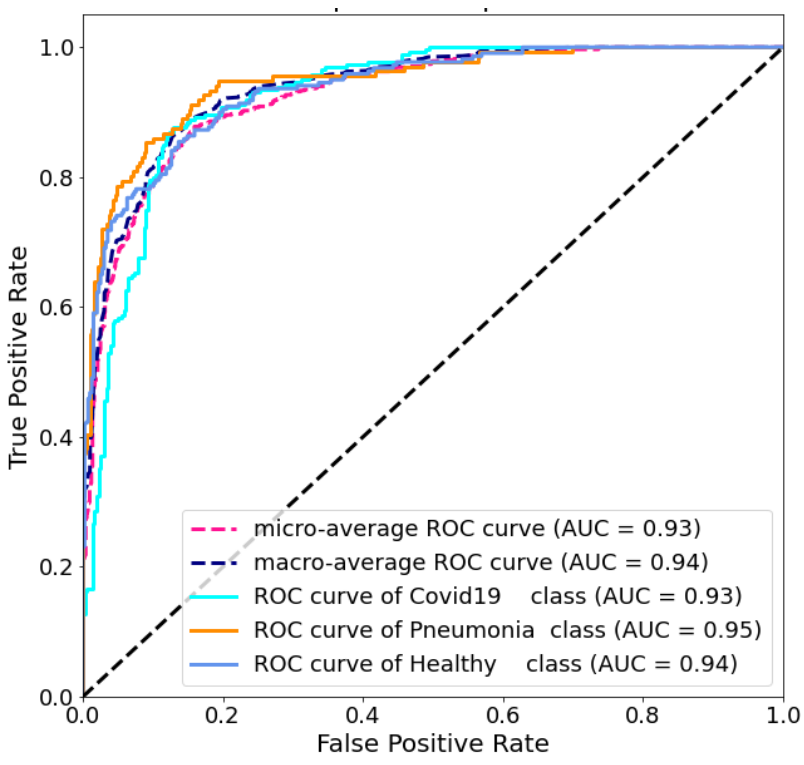

Fold3

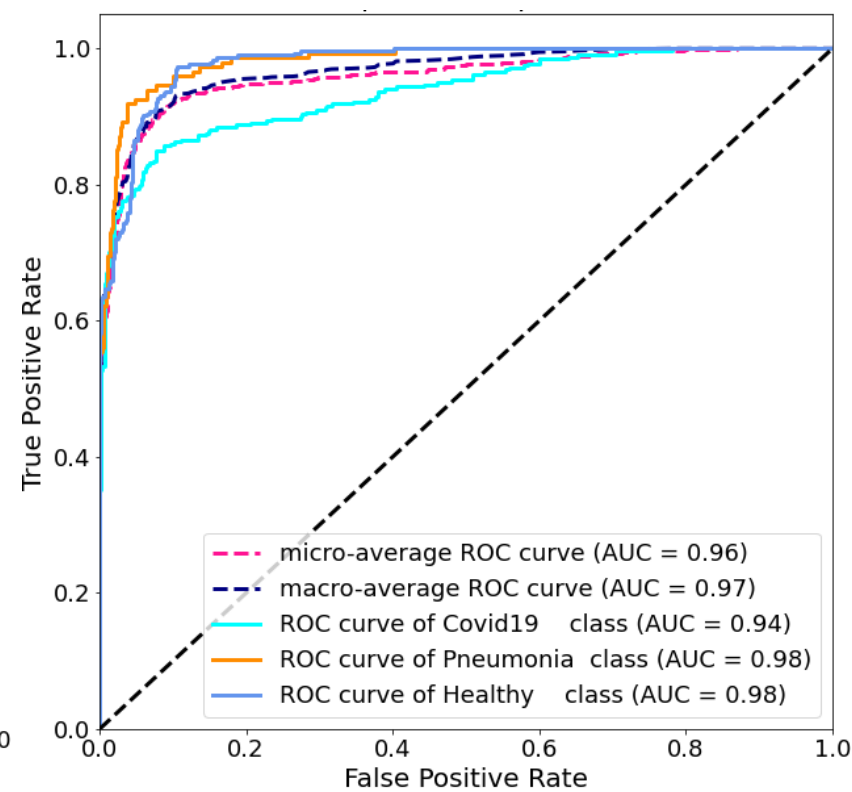




\section{$3 \times 5$-Fold Cross-Validation}

\section{Learning curves}

\section{Fold1}

Training Loss and Accuracy on COVID-19 Dataset

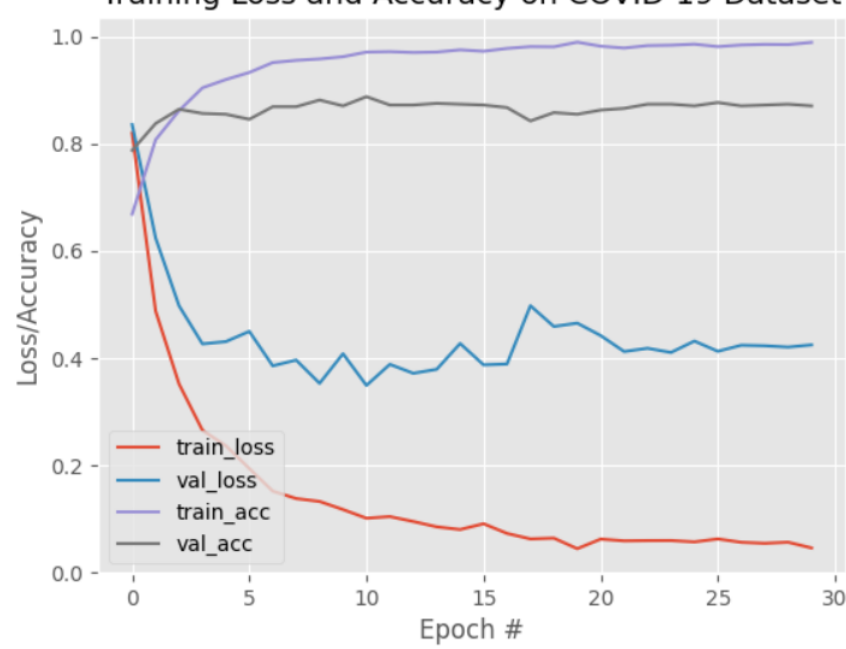

Fold4

Training Loss and Accuracy on COVID-19 Dataset

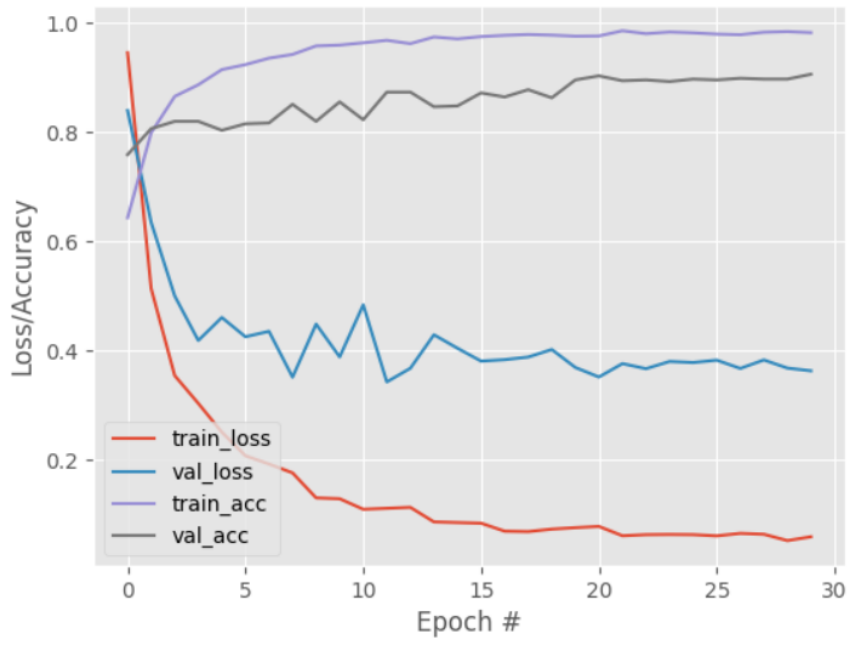

Fold2

Training Loss and Accuracy on COVID-19 Dataset

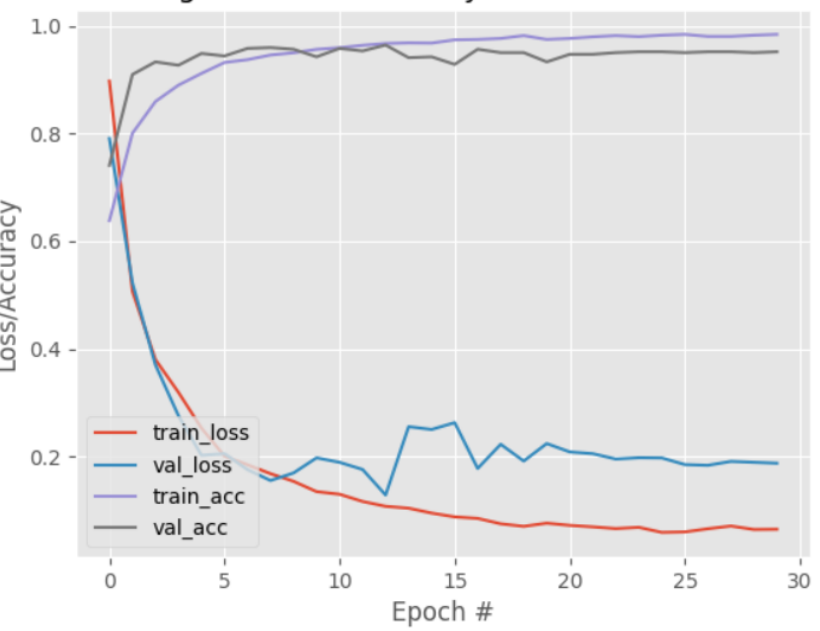

Fold5

Training Loss and Accuracy on COVID-19 Dataset

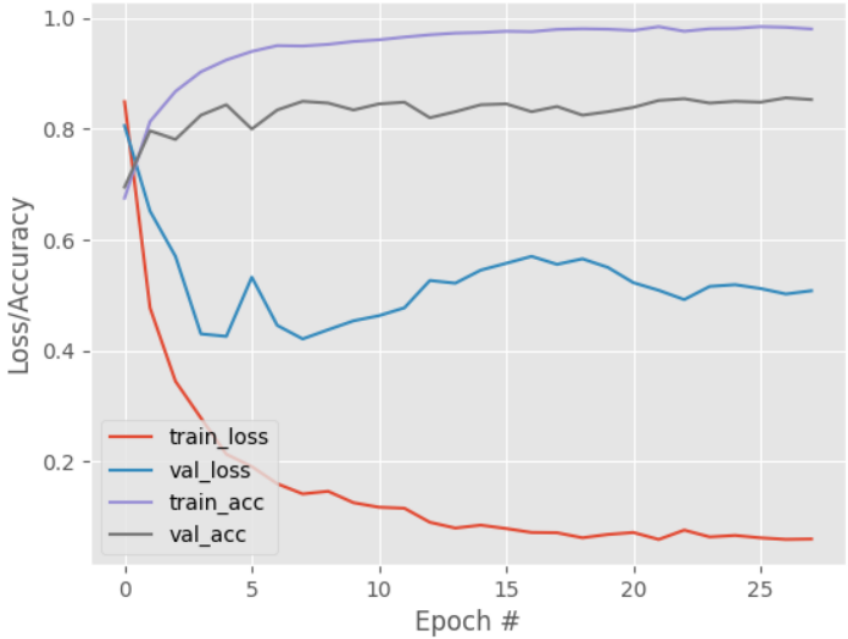

Fold3

Training Loss and Accuracy on COVID-19 Dataset

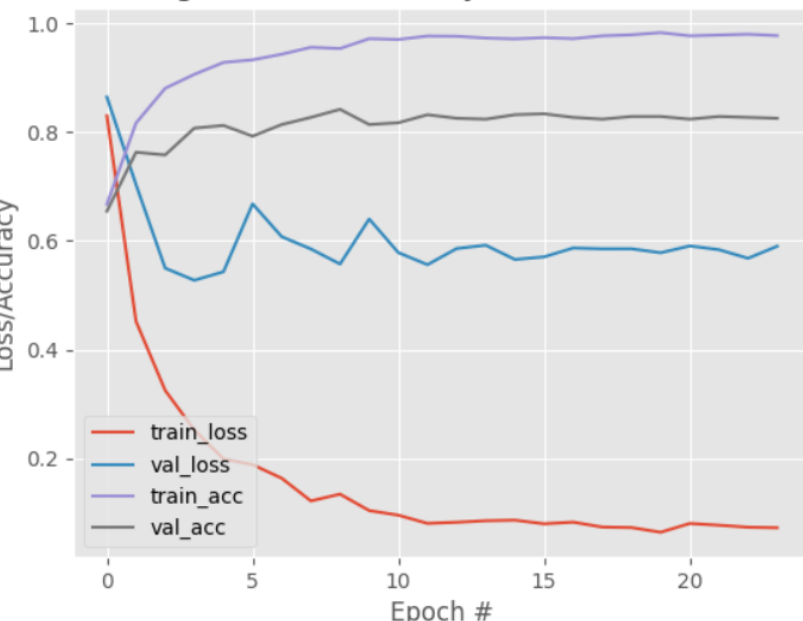




\section{Confusion matrices}

Fold1

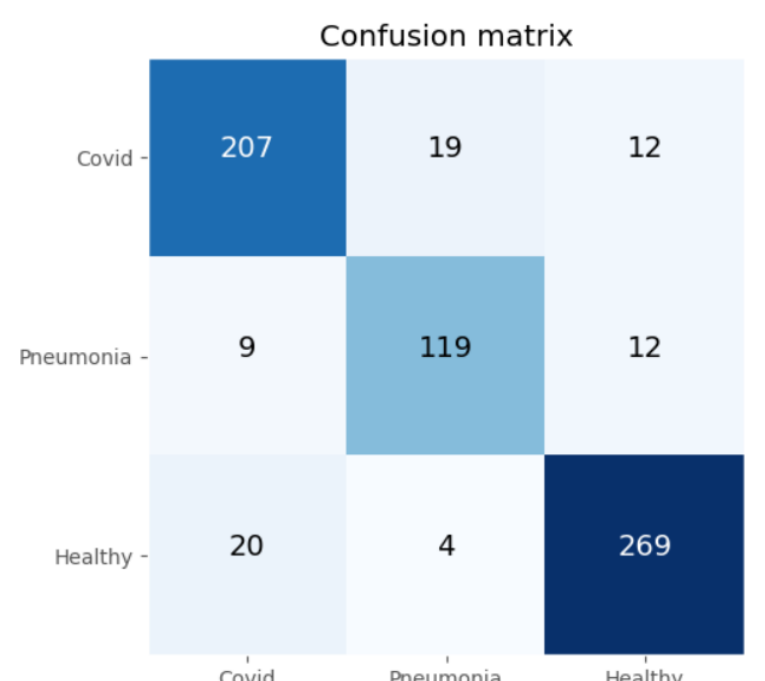

Covid Pneumonia Healthy

Fold4

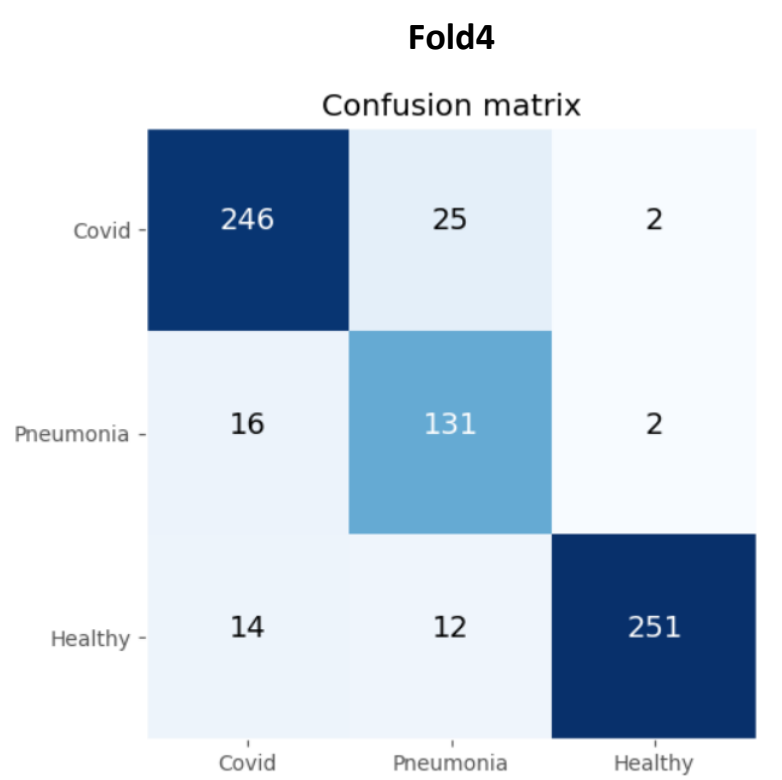

Fold2

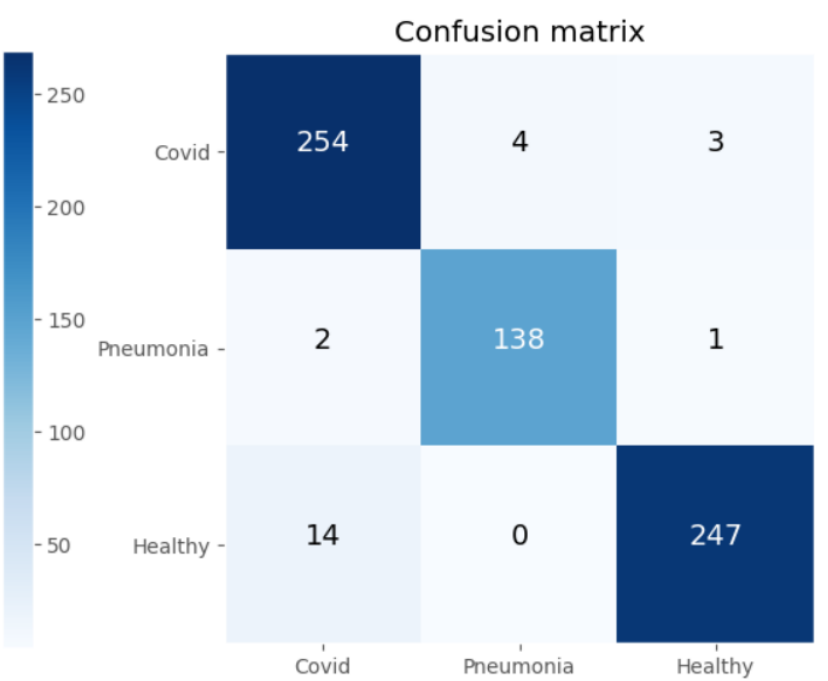

Fold5

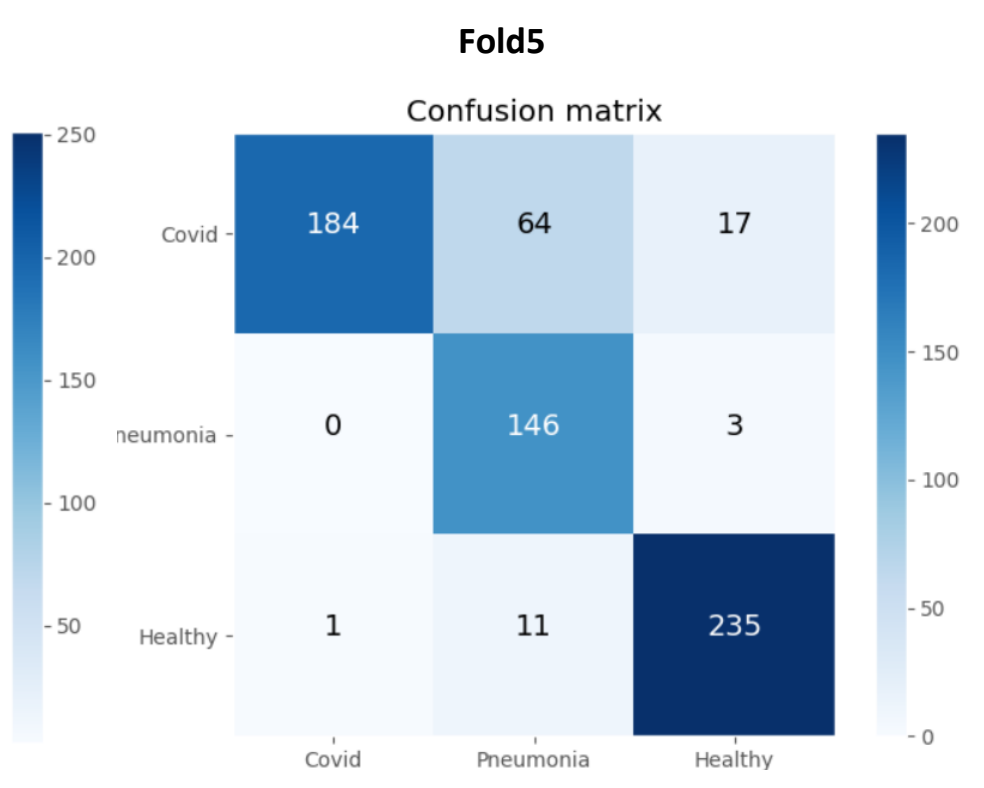

Fold3

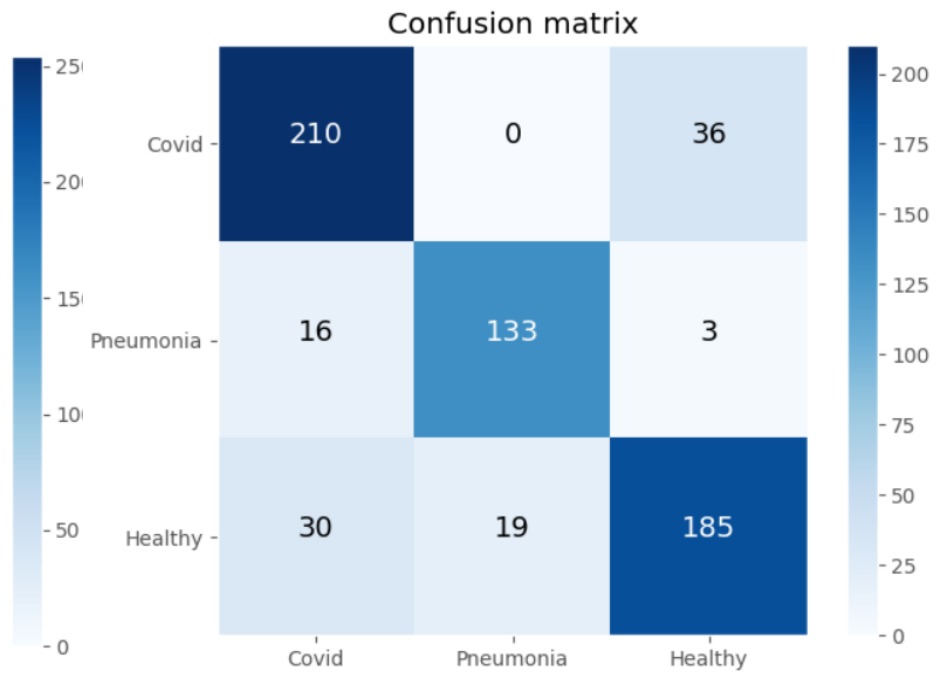


Fold1

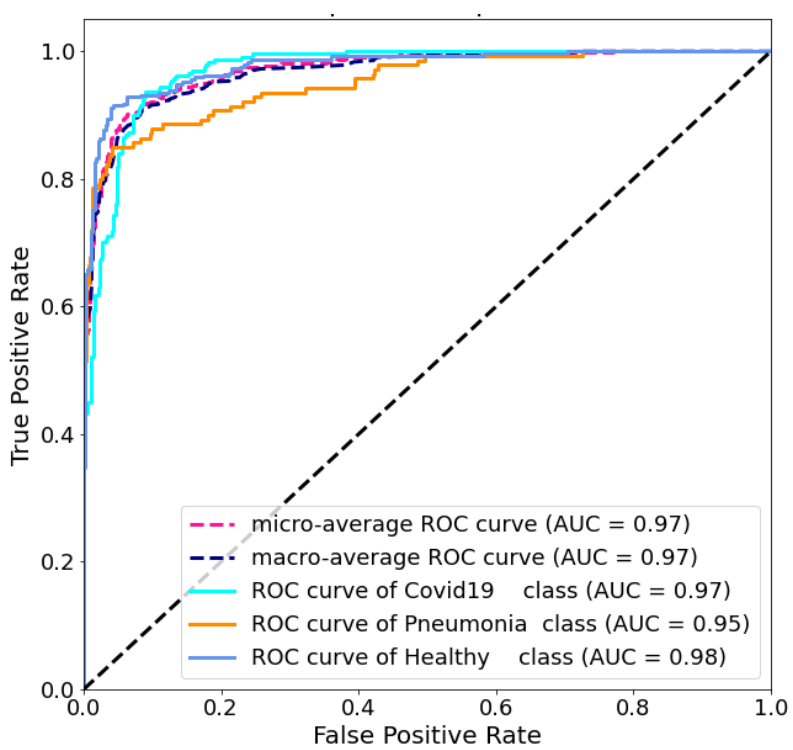

Fold4

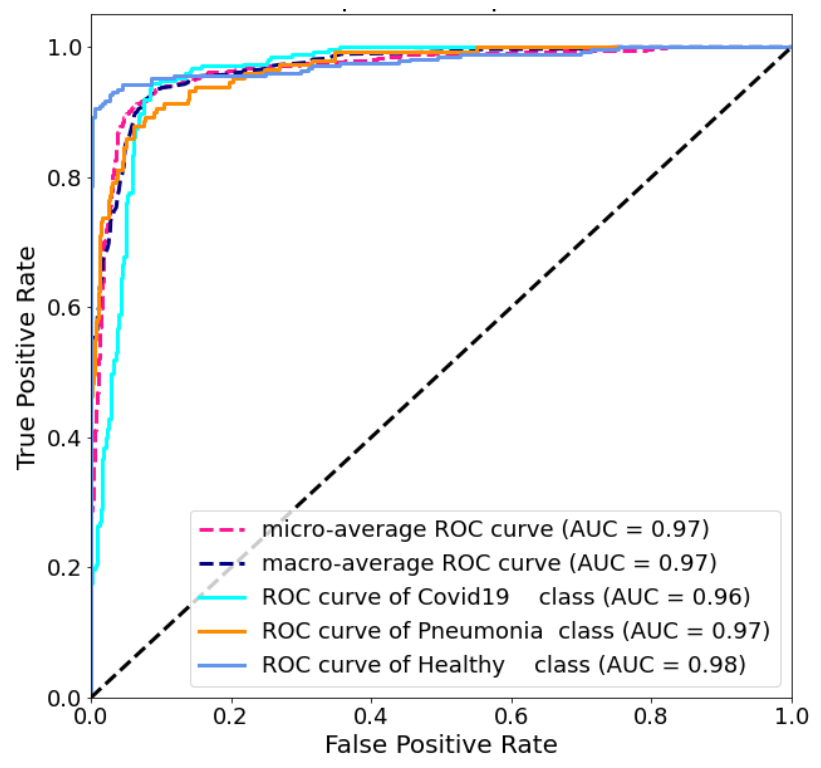

Fold2

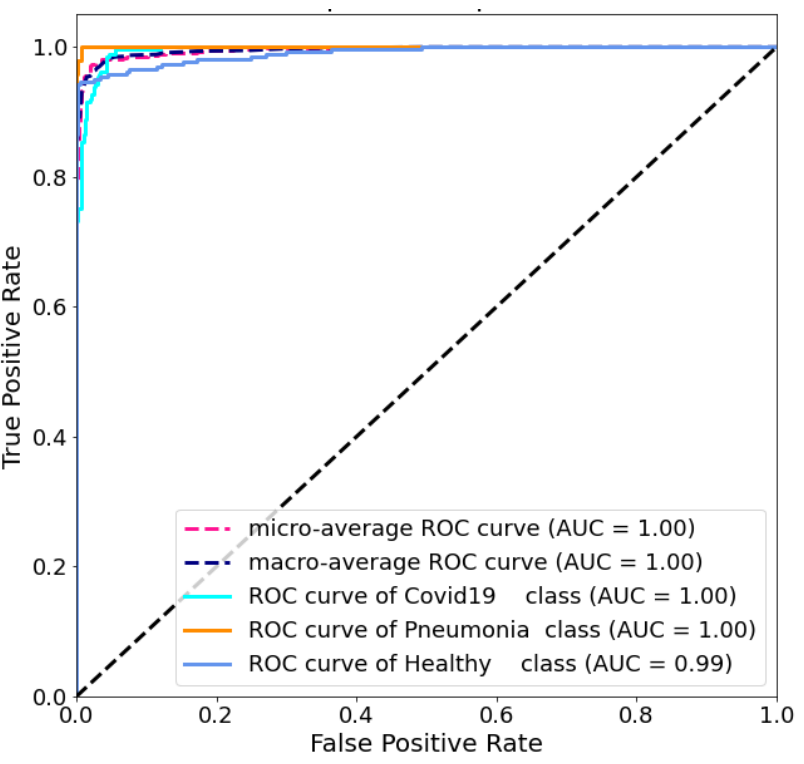

\section{Fold5}

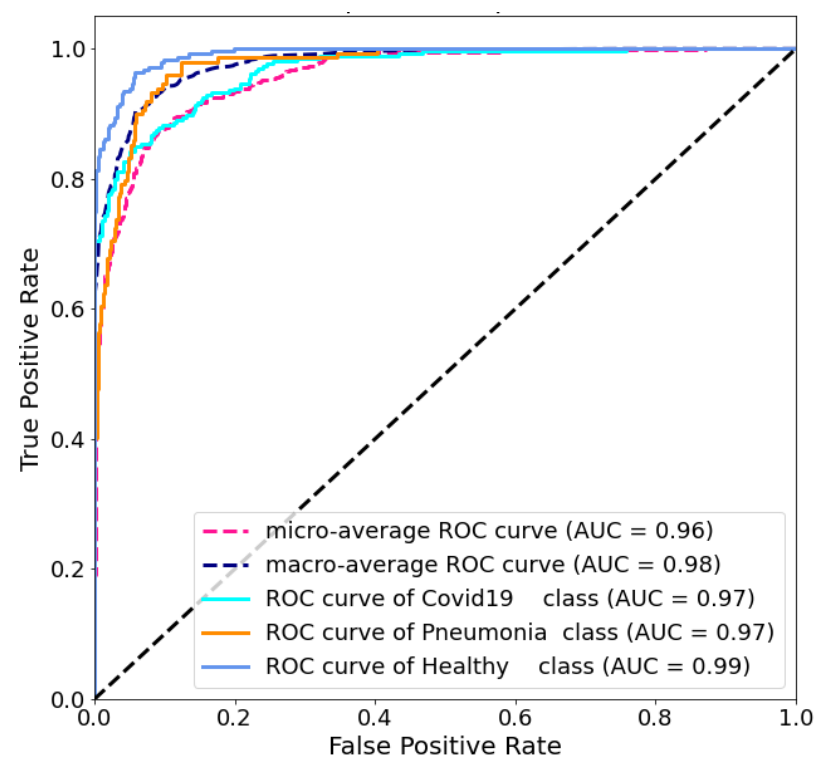

Fold3

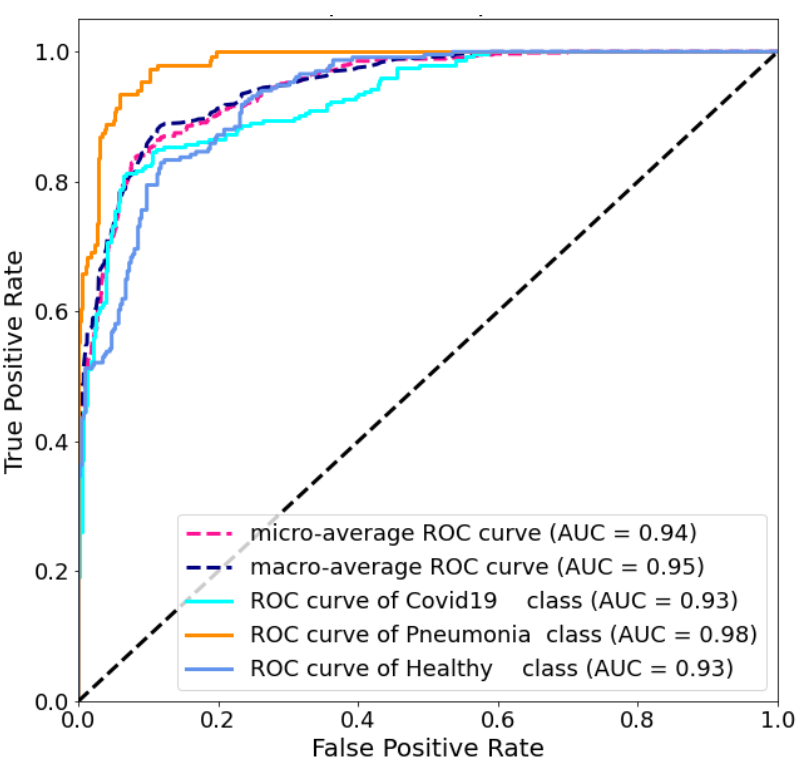




\section{$4 \times 5$-Fold Cross-Validation}

\section{Learning curves}

\section{Fold1}

Training Loss and Accuracy on COVID-19 Dataset

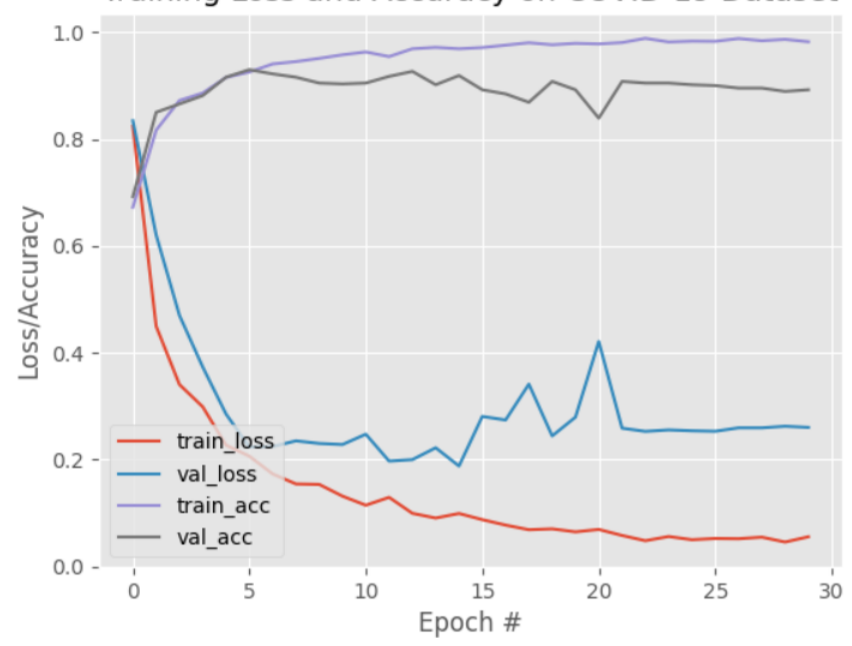

Fold4

Training Loss and Accuracy on COVID-19 Dataset

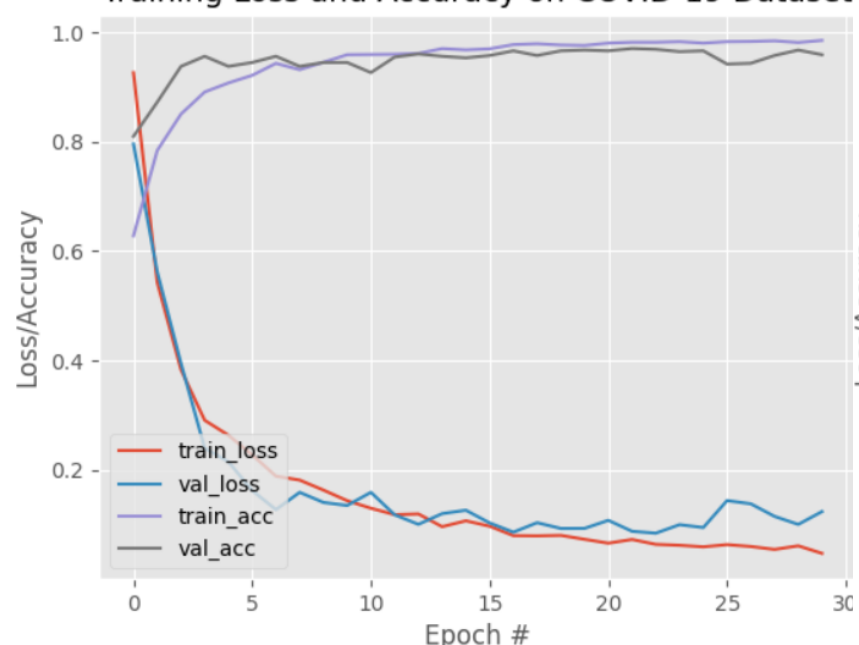

Fold2

Training Loss and Accuracy on COVID-19 Dataset

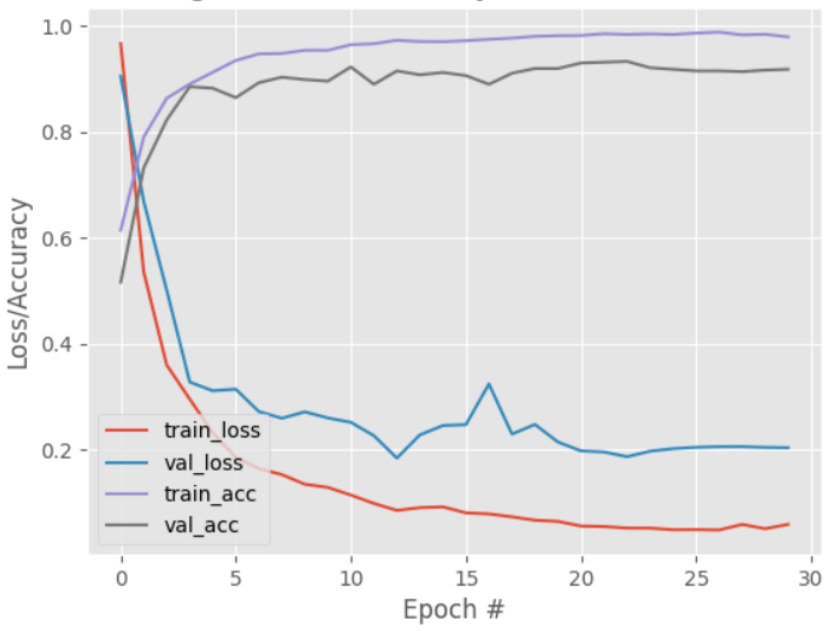

Fold5

Training Loss and Accuracy on COVID-19 Dataset

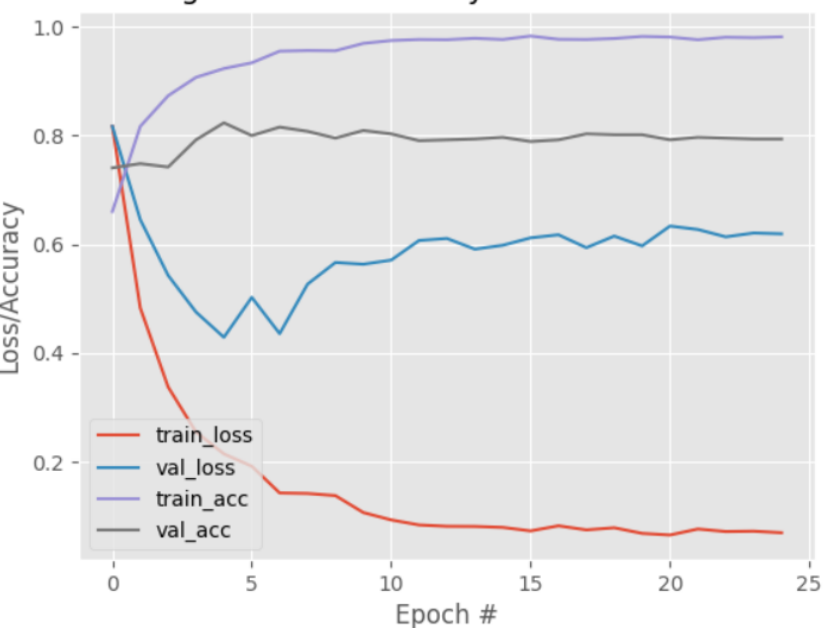

Fold3

Training Loss and Accuracy on COVID-19 Dataset

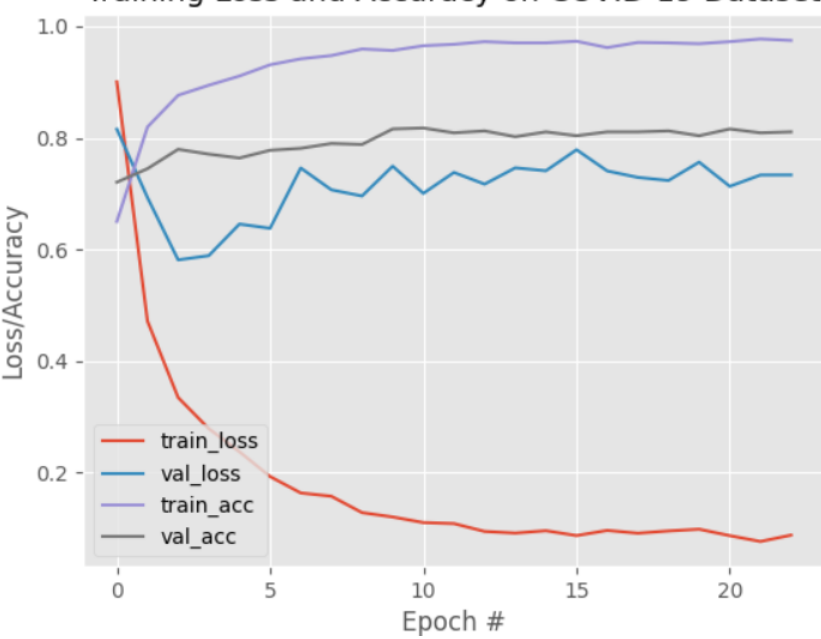




\section{Confusion matrices}

Fold1

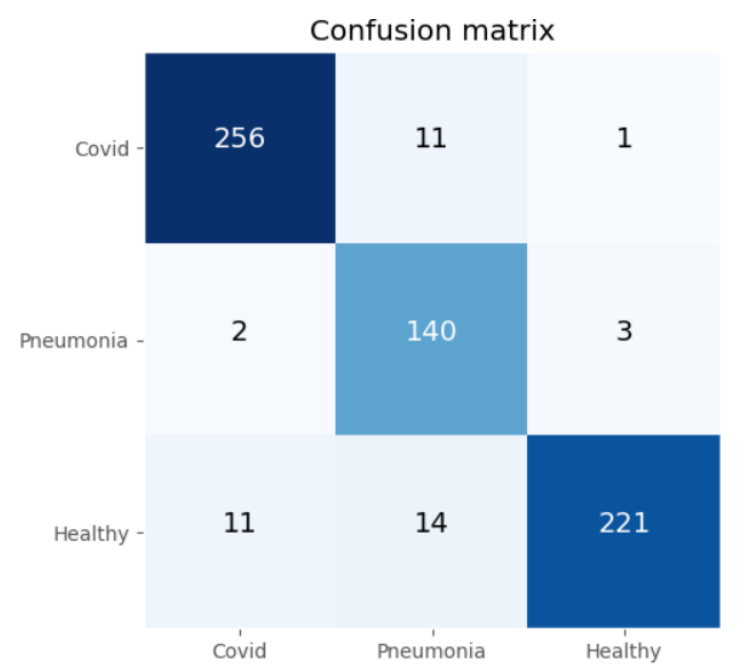

Fold4

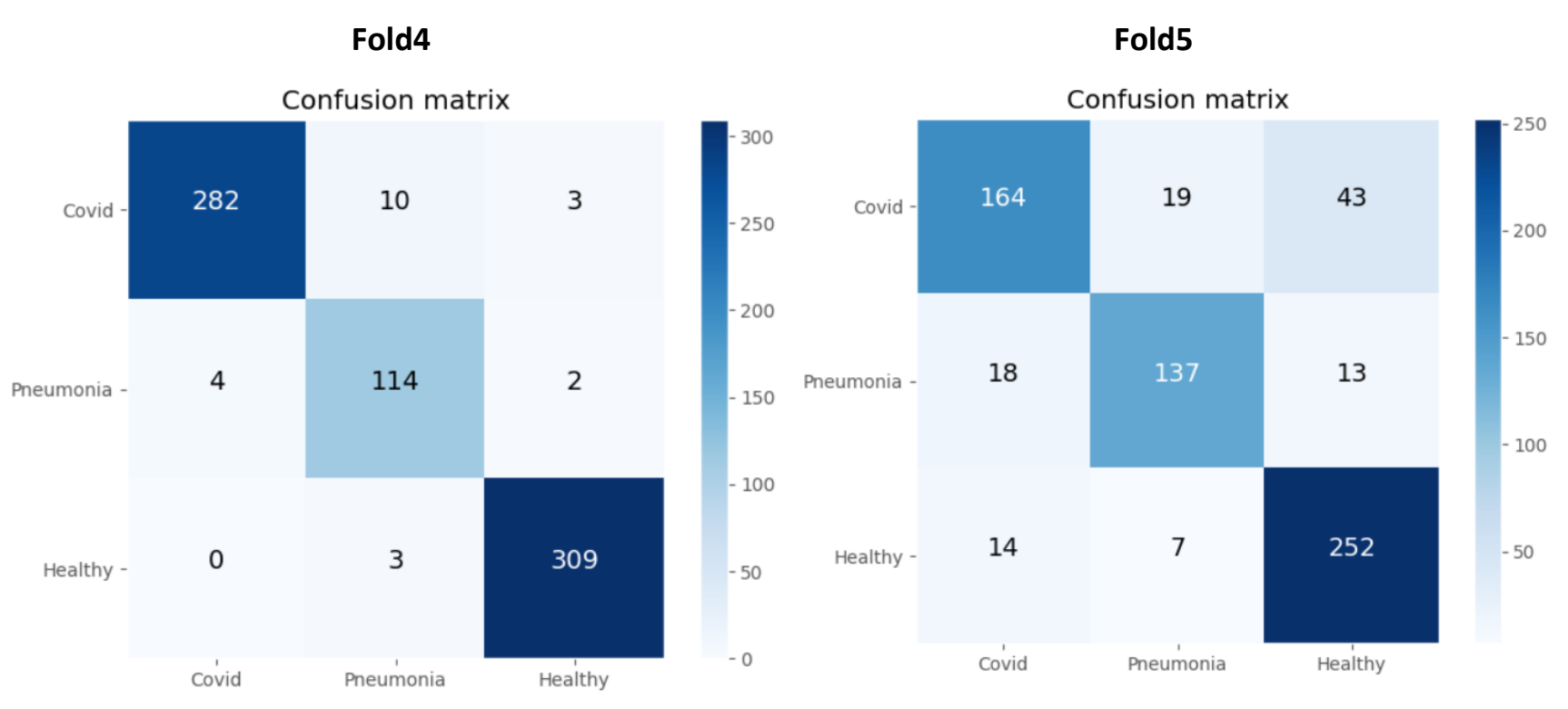

Fold2
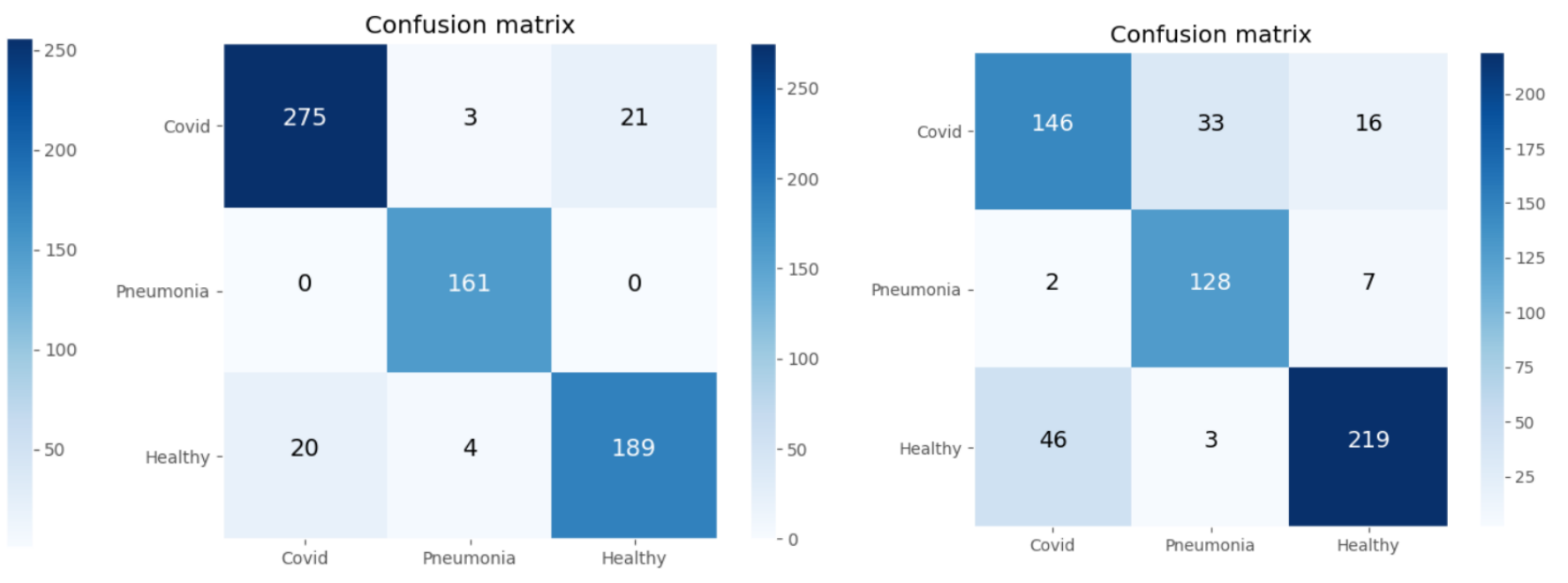
Fold1

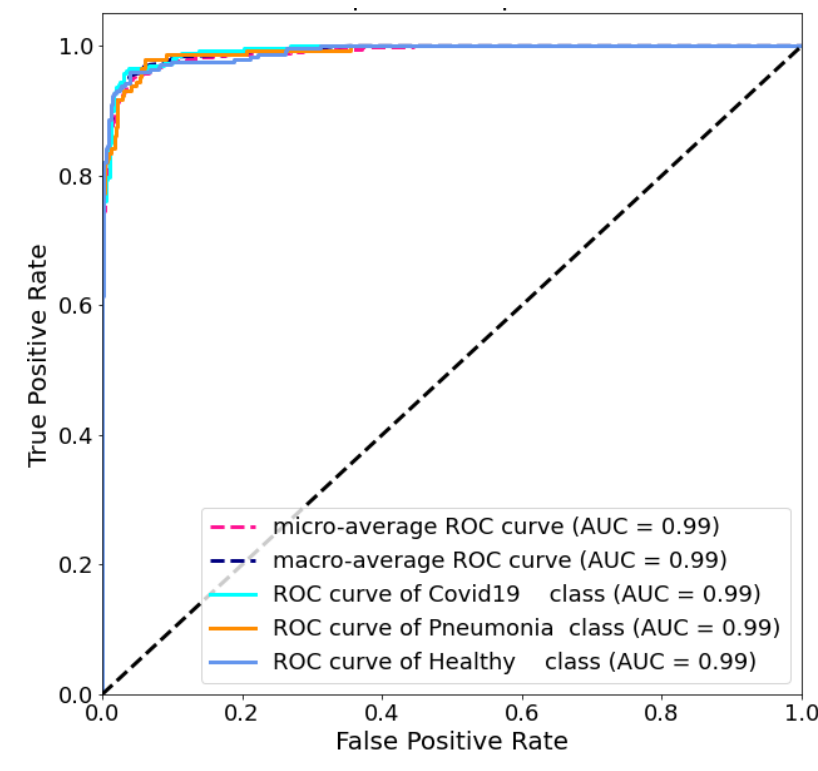

Fold4

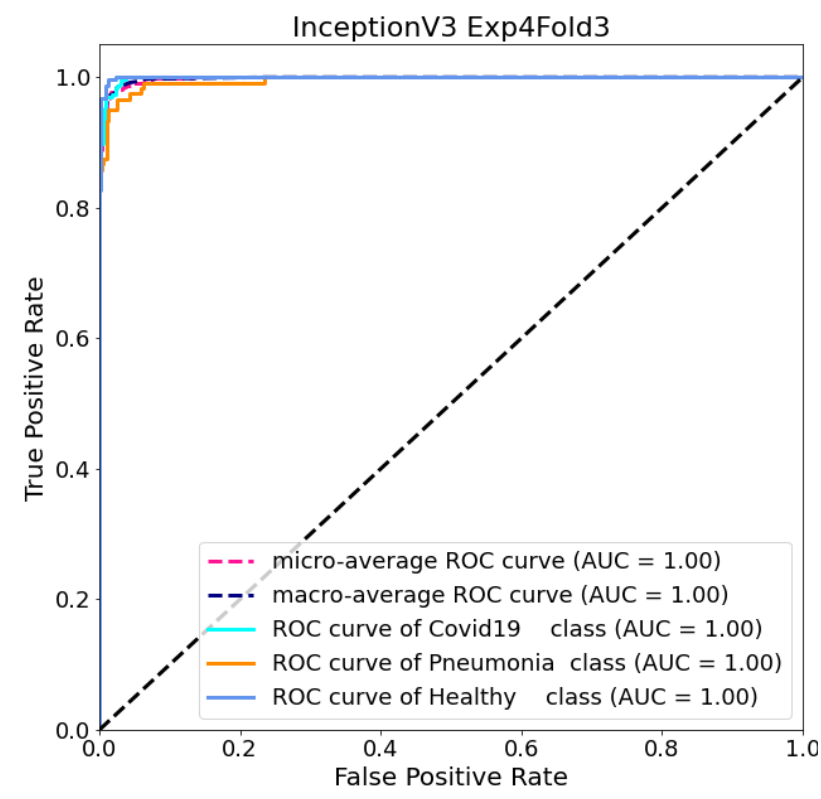

Fold2

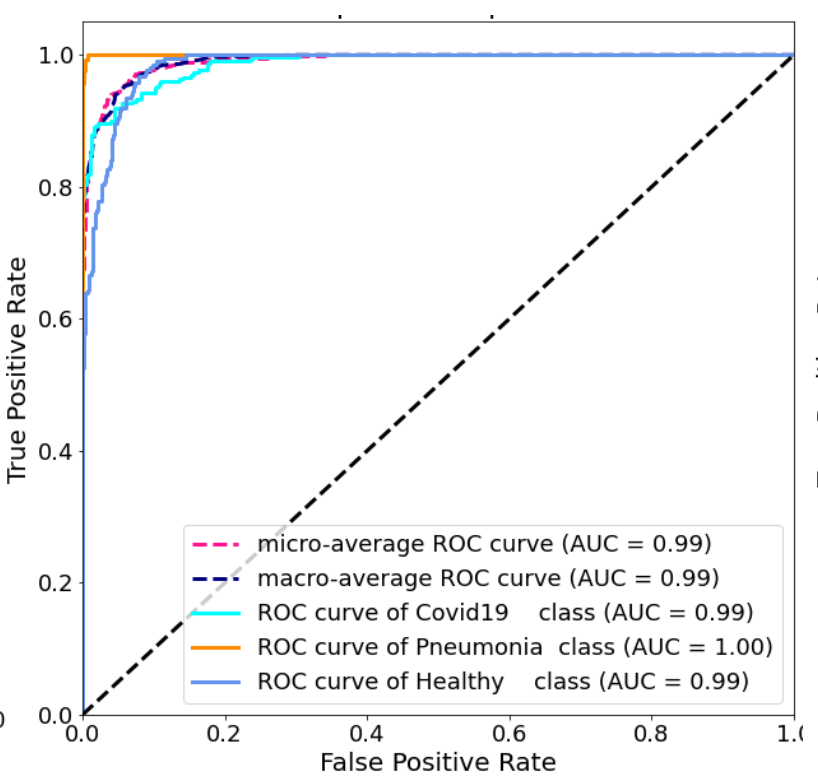

Fold5

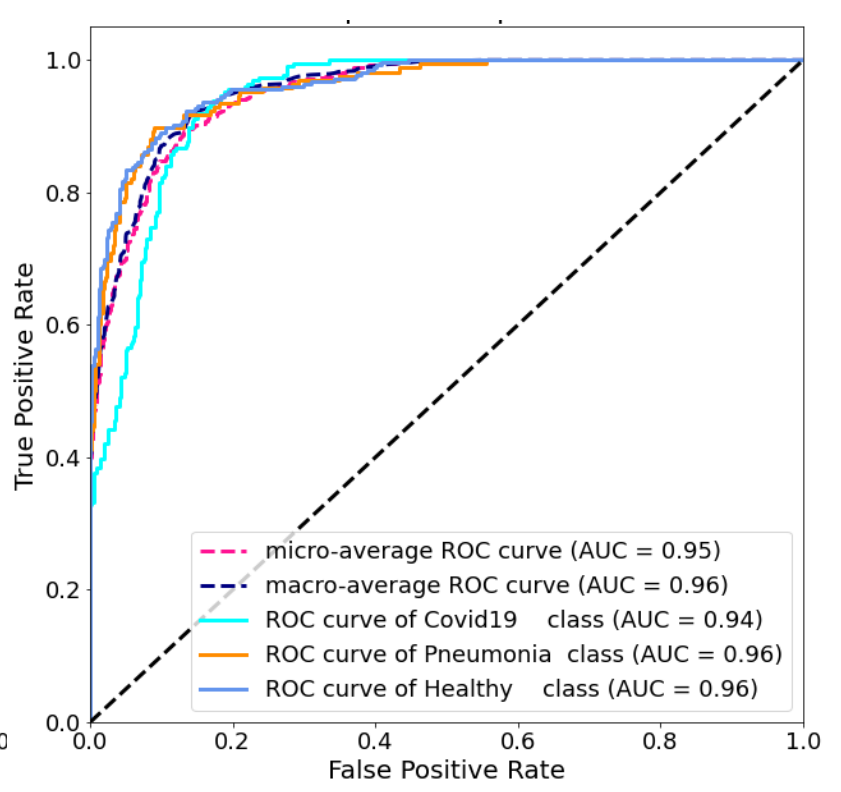

Fold3

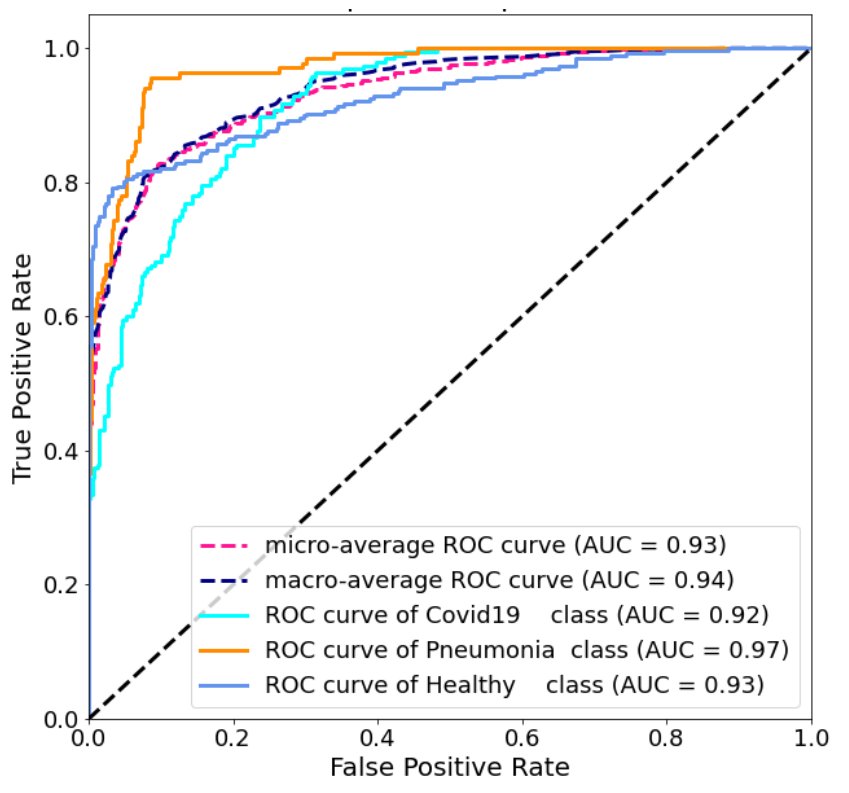




\section{$5 \times 5$-Fold Cross-Validation}

\section{Learning curves}

\section{Fold1}

Training Loss and Accuracy on COVID-19 Datase

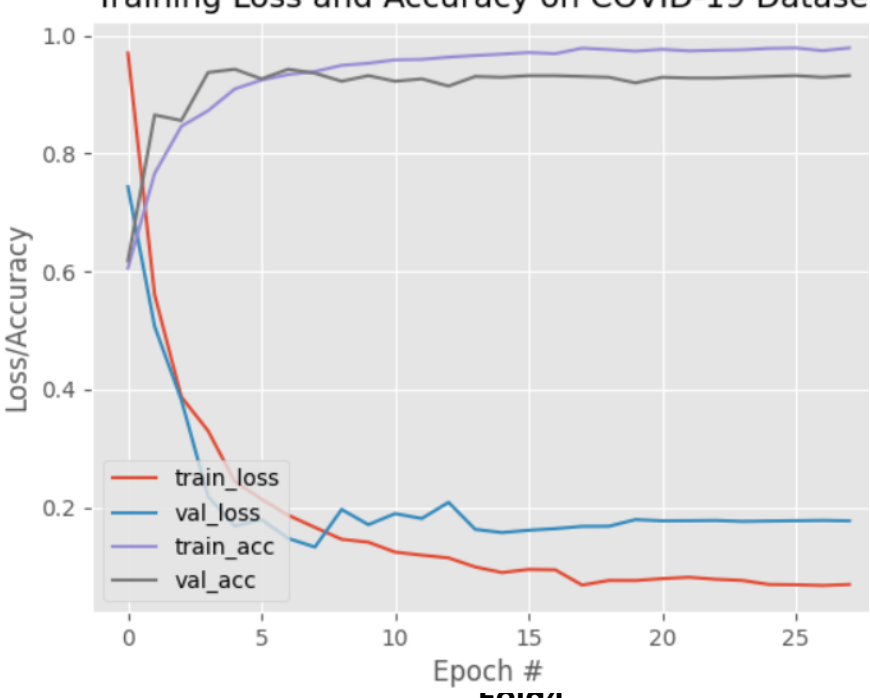

rola4

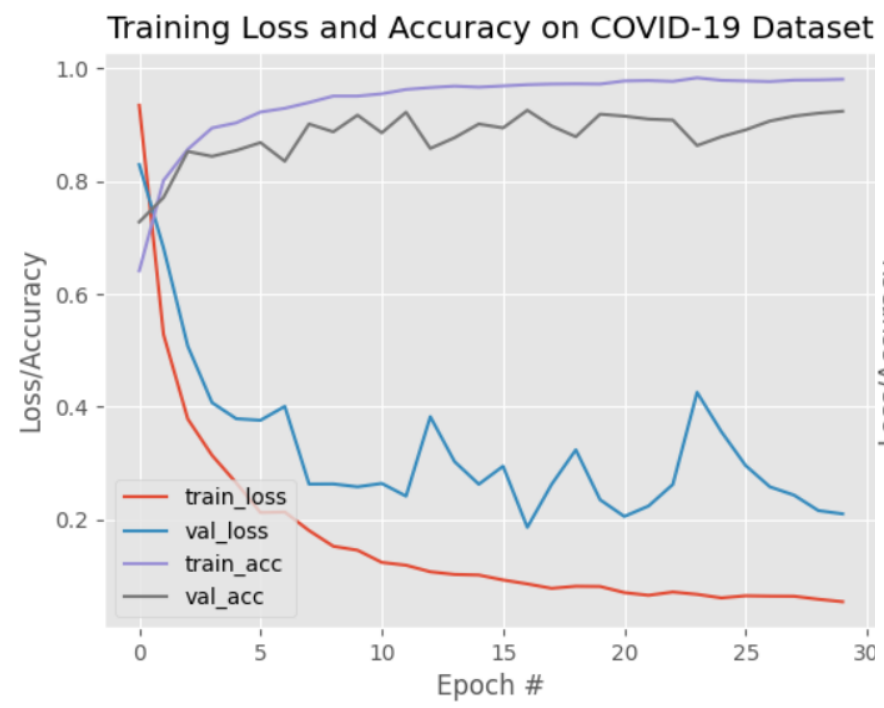

Fold2

Training Loss and Accuracy on COVID-19 Dataset

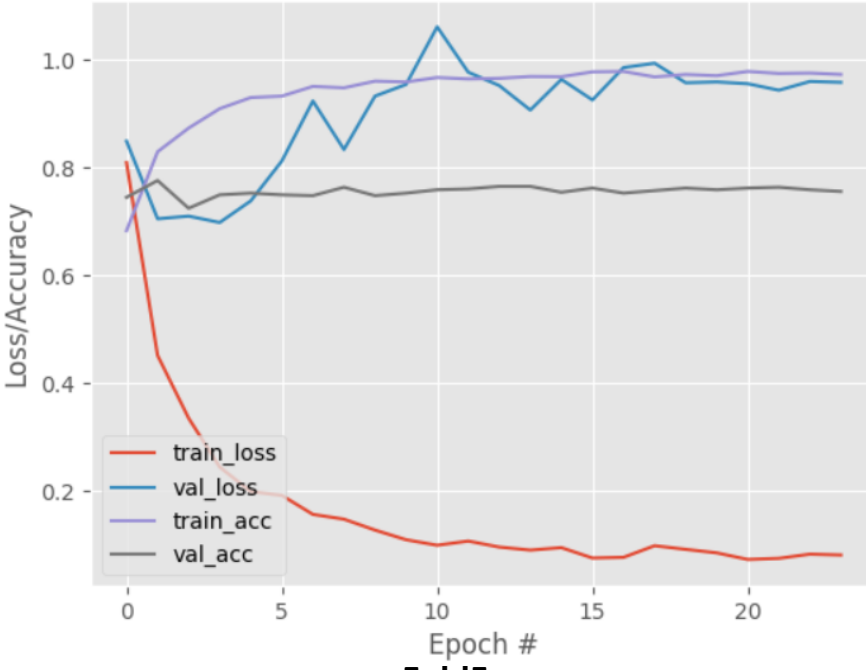

Fold5

Training Loss and Accuracy on COVID-19 Dataset

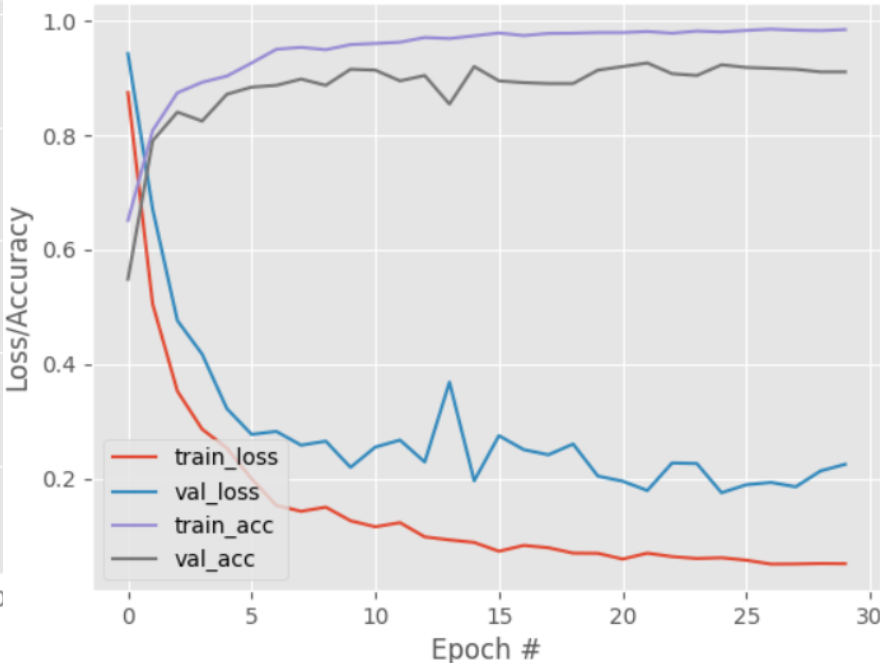

Fold3

Training Loss and Accuracy on COVID-19 Dataset

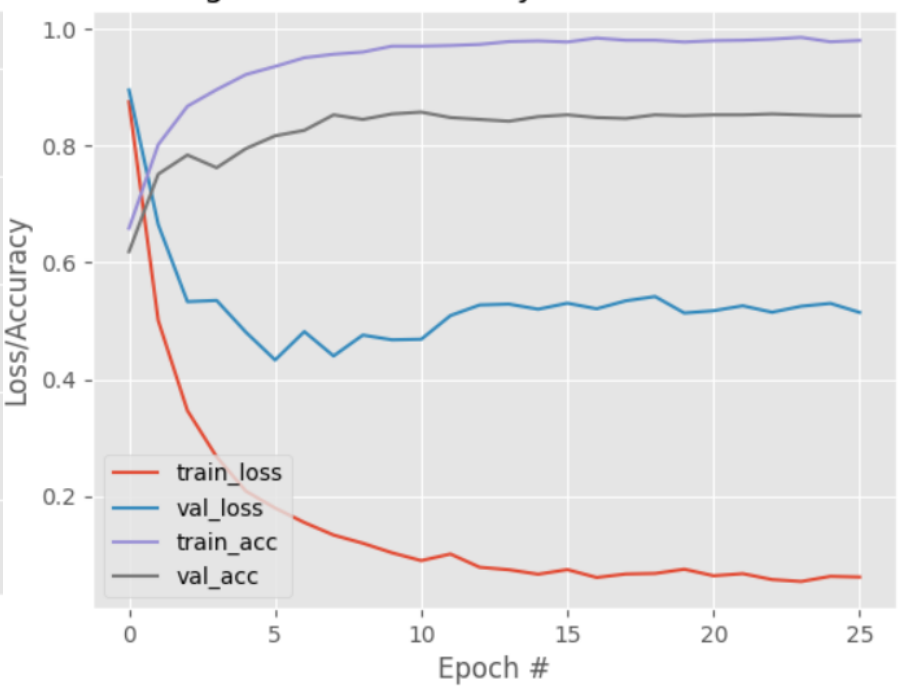




\section{Confusion matrices}

Fold1

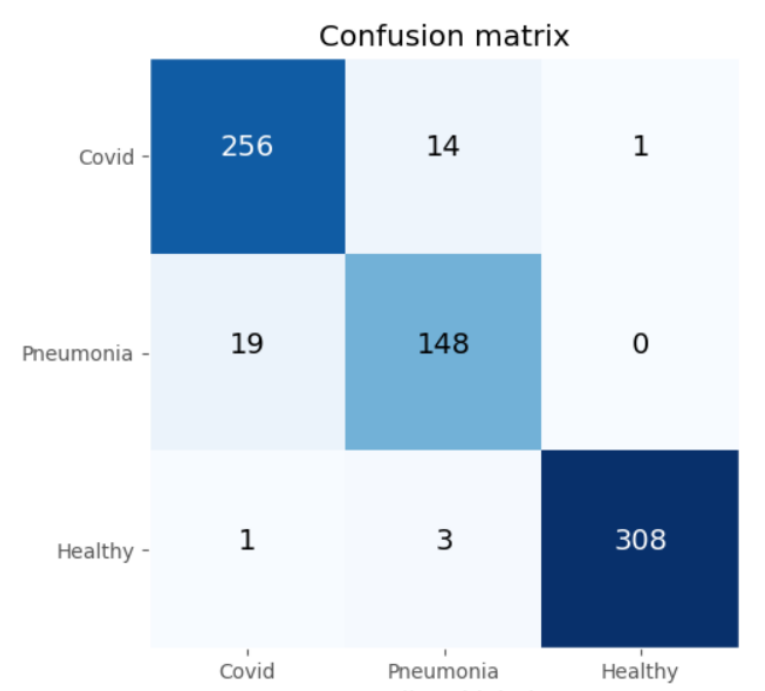

Fold4

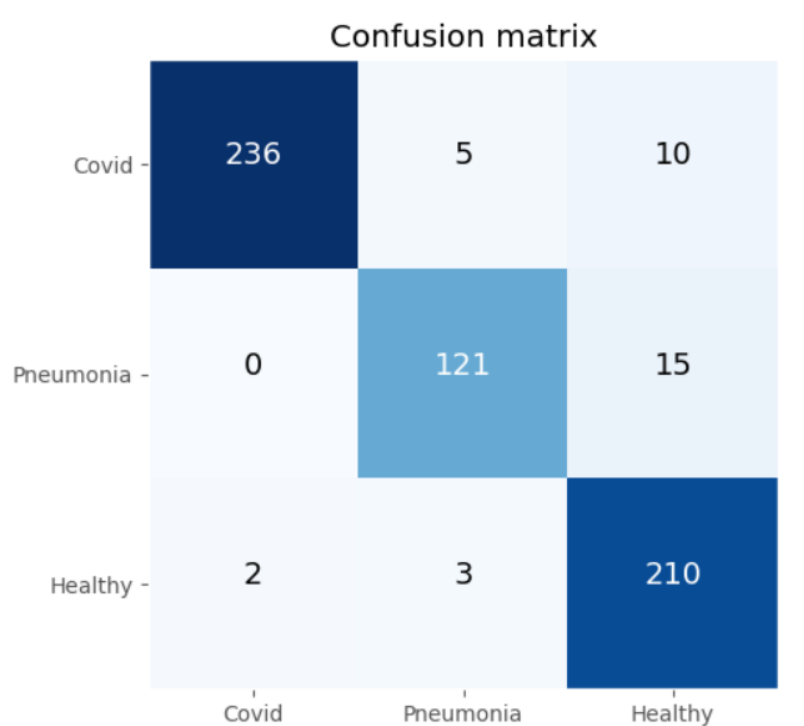

Fold2
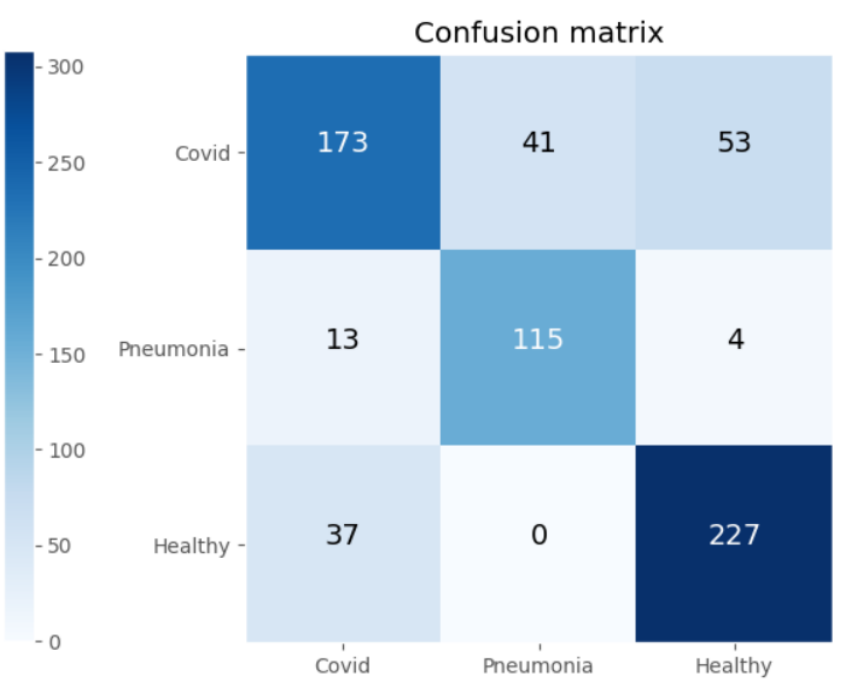

Fold5

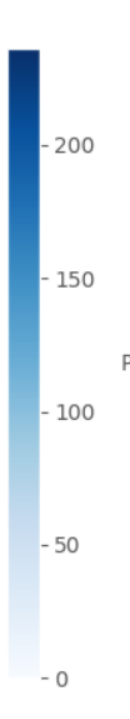

Confusion matrix
Fold3
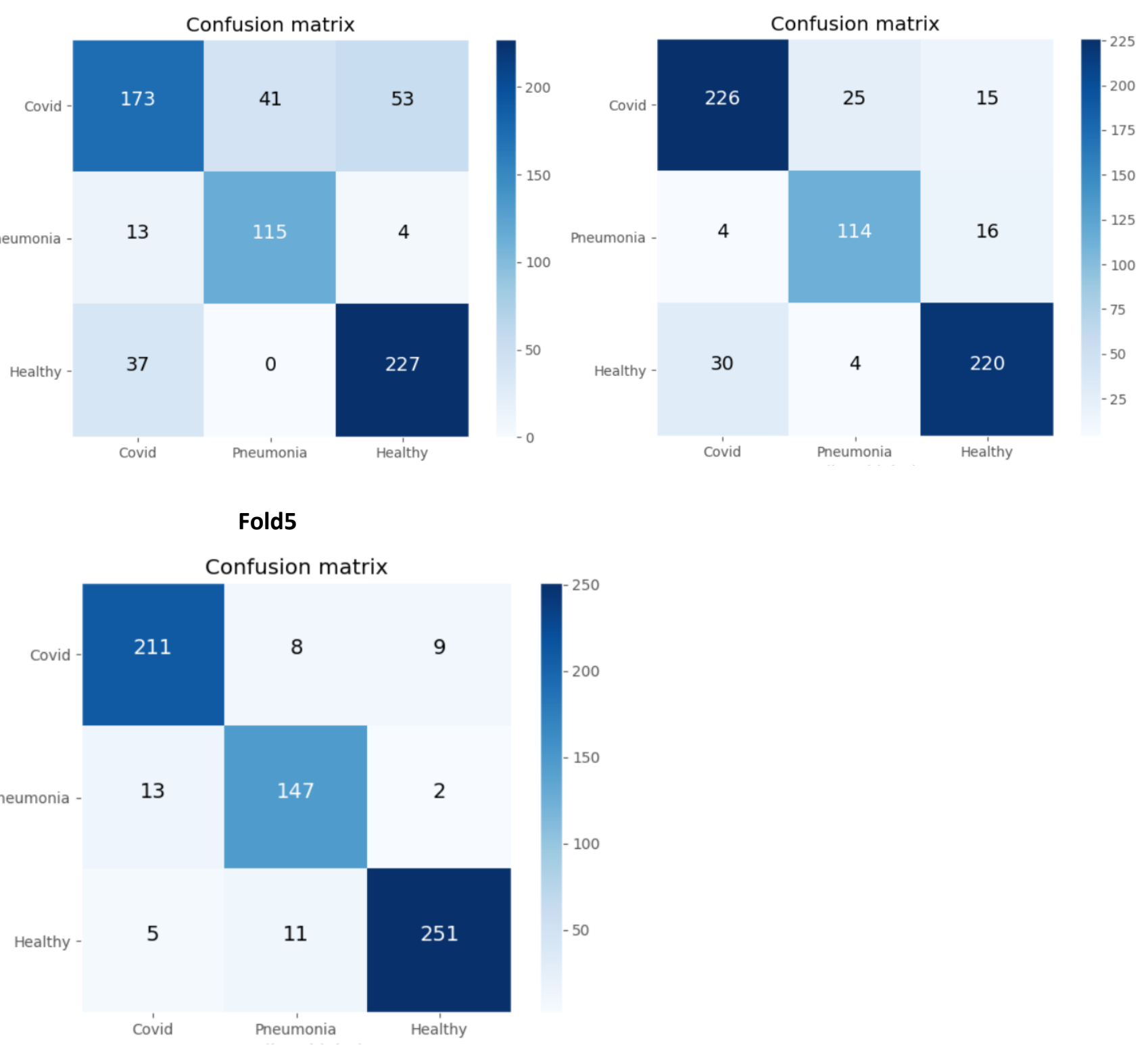
Fold1

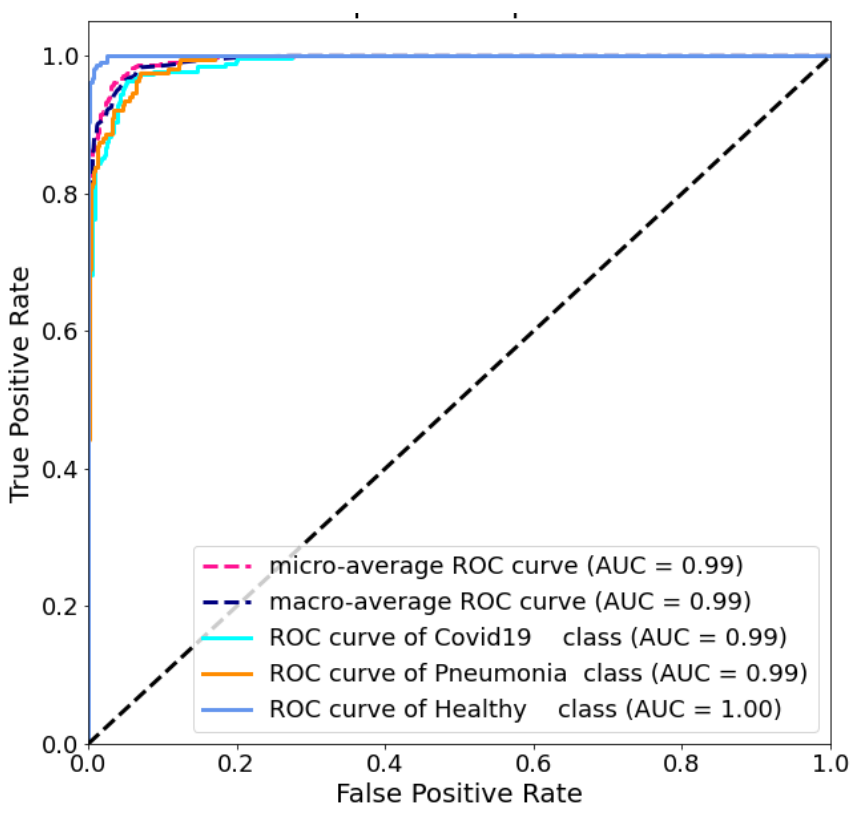

Fold4

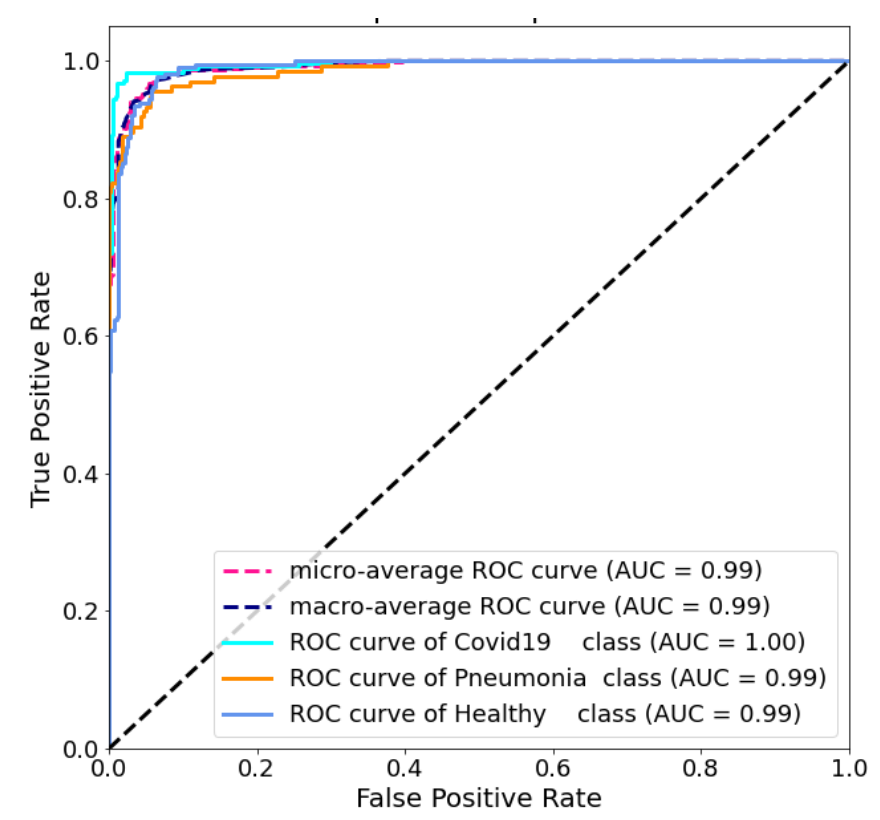

Fold2

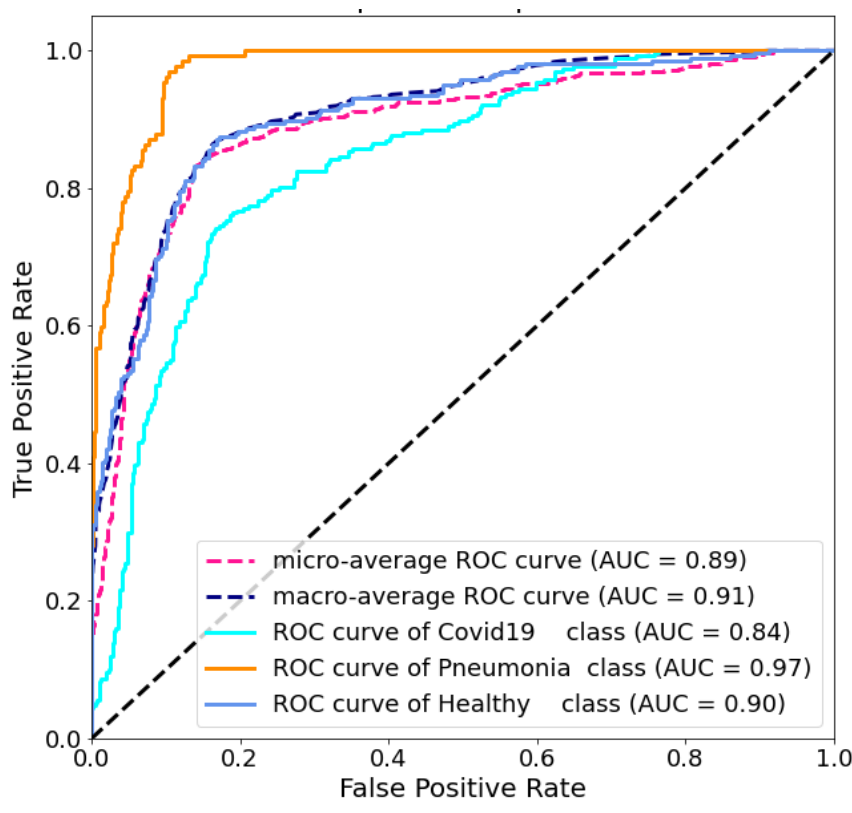

Fold5

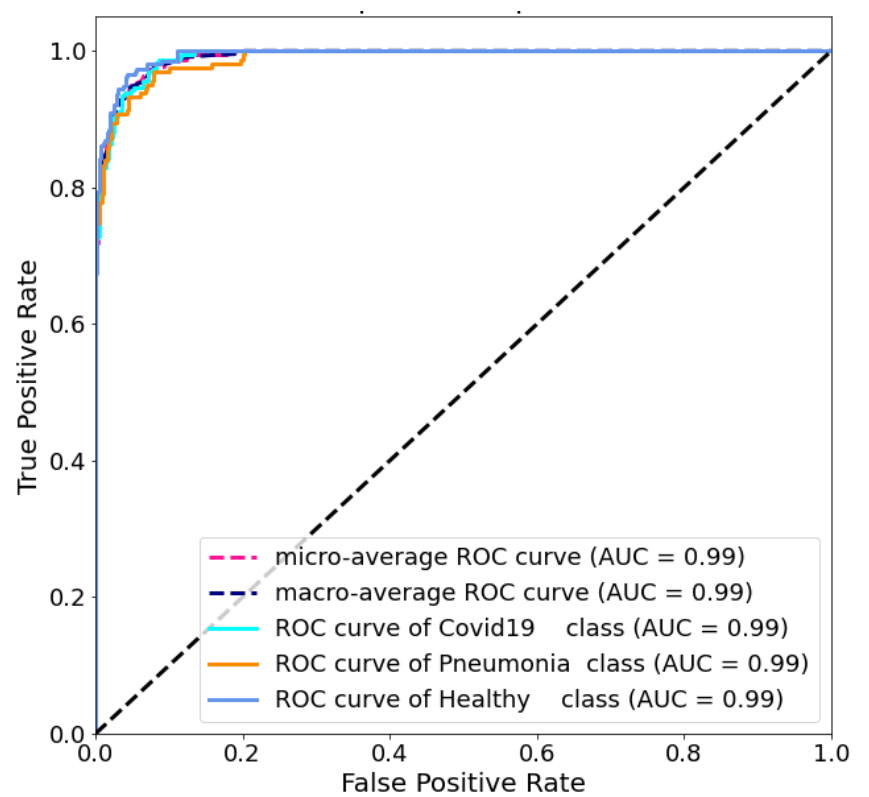

Fold3

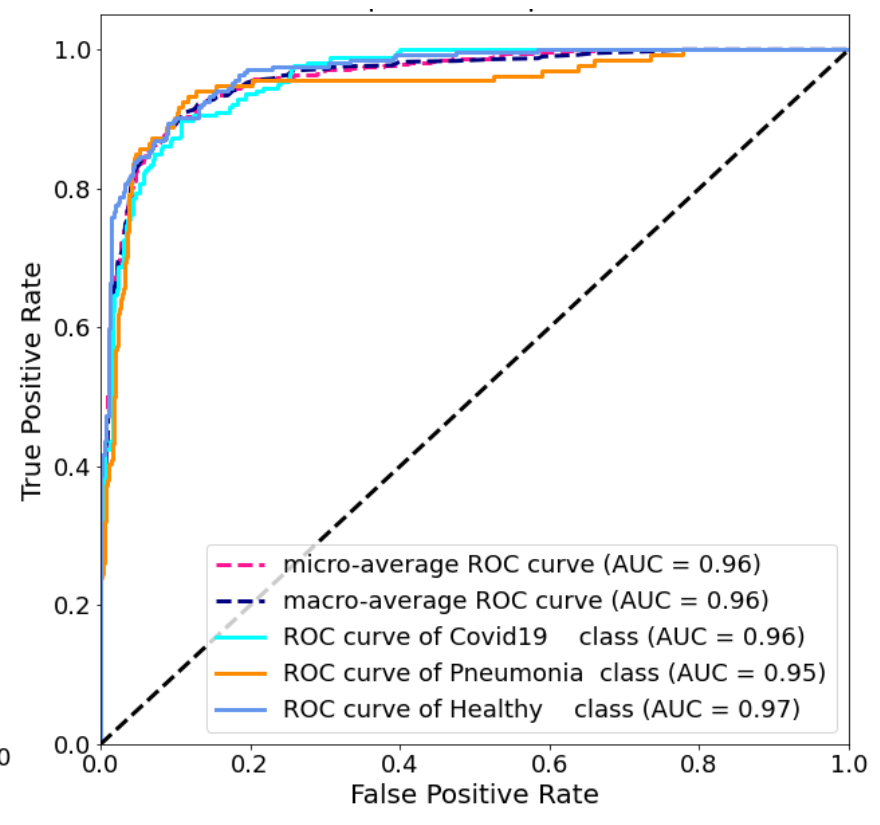

